\title{
Clinical Imaging
}

\section{Benign and malignant mimickers of infiltrative hepatocellular carcinoma: tips and tricks for differential diagnosis on CT and MRI \\ --Manuscript Draft--}

\begin{tabular}{|c|c|}
\hline Manuscript Number: & \\
\hline Article Type: & Review Article \\
\hline Keywords: & $\begin{array}{l}\text { Hepatocellular carcinoma; Liver neoplasms; Differential diagnosis; Computed } \\
\text { tomography; Magnetic Resonance Imaging }\end{array}$ \\
\hline Corresponding Author: & $\begin{array}{l}\text { Federica Vernuccio } \\
\text { ITALY }\end{array}$ \\
\hline First Author: & Federica Vernuccio \\
\hline Order of Authors: & Federica Vernuccio \\
\hline & Giorgia Porrello \\
\hline & Roberto Cannella \\
\hline & Laura Vernuccio \\
\hline & Massimo Midiri \\
\hline & Lydia Giannitrapani \\
\hline & Maurizio Soresi \\
\hline & Giuseppe Brancatelli \\
\hline Abstract: & $\begin{array}{l}\text { Hepatocellular carcinoma (HCC) may have an infiltrative appearance in about } 8-20 \% \\
\text { of cases. Infiltrative HCC can be a challenging diagnosis and it is associated with the } \\
\text { worst overall survival among HCC patients. Infiltrative HCC is characterized by the } \\
\text { spread of multiple minute nodules throughout the liver, without a dominant one, } \\
\text { ultimately determining macrovascular invasion. On CT and MRI, infiltrative HCC } \\
\text { appears as an ill-defined, large mass, with variable degree of enhancement, and } \\
\text { satellite neoplastic nodules in up to } 52 \% \text { of patients. On MRI, it may show restriction on } \\
\text { diffusion weighted imaging, hyperintensity on T2- and hypointensity on T1-weighted } \\
\text { images, and, if hepatobiliary agent is used, hypointensity on hepatobiliary phase. } \\
\text { Infiltrative HCC must be differentiated from other liver diseases, such as focal confluent } \\
\text { fibrosis, steatosis, amyloidosis, vascular disorders of the liver, cholangiocarcinoma, } \\
\text { and diffuse metastatic disease. In cirrhotic patients, the identification of vascular tumor } \\
\text { invasion of the portal vein and its differentiation from bland thrombosis is of utmost } \\
\text { importance for patient management. On contrast enhanced CT and MRI portal vein } \\
\text { tumor thrombosis appears as an enhancing thrombus within the portal vein, close to } \\
\text { the main tumor and determines vein enlargement. The aim of this pictorial review is to } \\
\text { show CT and MRI features that allow the diagnosis of infiltrative HCC and portal vein } \\
\text { tumor thrombosis. A particular point of interest includes the tips and tricks for } \\
\text { differential diagnosis with potential mimickers of infiltrative HCC. }\end{array}$ \\
\hline Suggested Reviewers: & $\begin{array}{l}\text { Kalina Chupetlovska } \\
\text { k.chupetlovska@outlook.com }\end{array}$ \\
\hline & $\begin{array}{l}\text { Alessandro Furlan } \\
\text { furlana@upmc.edu }\end{array}$ \\
\hline
\end{tabular}




\section{Benign and malignant mimickers of infiltrative hepatocellular carcinoma: tips and tricks for differential diagnosis on CT and MRI}

Federica Vernuccio, MD1,2,3,4; Giorgia Porrello4, Roberto Cannella ${ }^{4}$, Laura Vernuccio ${ }^{5}$, Massimo Midiri ${ }^{4}$, Lydia Giannitrapani ${ }^{1}$, Maurizio Soresi ${ }^{1}$, Giuseppe Brancatelli ${ }^{4}$

1. Department of Health Promotion, Mother and Child Care, Internal Medicine and Medical Specialties (PROMISE),, University of Palermo, Palermo, Italy

2. University Paris Diderot, Sorbonne Paris Cité, Paris, France;

3. I.R.C.C.S. Centro Neurolesi Bonino Pulejo, Contrada Casazza, SS113, 98124 Messina

4. Department of Biomedicine, Neuroscience and Advanced Diagnostics (BIND), University Hospital of Palermo, Via del Vespro 129, 90127 Palermo, Italy.

5. U.O.C. Geriatria e Lungodegenza, AOUP University Hospital Palermo; CDCD Geriatria, University Hospital Palermo, Palermo .

Federica Vernuccio: federicavernuccio@gmail.com - federica.vernuccio@unipa.it Giorgia Porrello: giorgia.porrello@gmail.com

Roberto Cannella: rob.cannella89@gmail.com

Laura Vernuccio: la.vernuccio@gmail.com

Massimo Midiri: massimo.midiri@unipa.it

Lydia Giannitrapani: Iydiagiannitp@gmail.com

Maurizio Soresi: maurizio.soresi@unipa.it

Giuseppe Brancatelli: gbranca@yahoo.com

Corresponding Author: Federica Vernuccio, MD, Radiologist, PhD Fellow University of Palermo

Via del Vespro, 129

90127 - Palermo

Phone: +39 (388) 6332212

Email: federicavernuccio@gmail.com

Funding: None 


\section{Disclosures:}

The authors have the following disclosures:

Federica Vernuccio: nothing to disclose for this study;

Giorgia Porrello: nothing to disclose for this study;

Roberto Cannella: nothing to disclose for this study;

Laura Vernuccio: nothing to disclose for this study;

Massimo Midiri: nothing to disclose for this study;

Lydia Giannitrapani: nothing to disclose for this study;

Maurizio Soresi: nothing to disclose for this study;

Giuseppe Brancatelli: nothing to disclose for this study. 


\title{
Benign and malignant mimickers of infiltrative hepatocellular carcinoma: tips and tricks for differential diagnosis on CT and MRI
}

\author{
Pictorial Essay
}

\section{Abstract}

Hepatocellular carcinoma (HCC) may have an infiltrative appearance in about $8-20 \%$ of cases. Infiltrative HCC can be a challenging diagnosis and it is associated with the worst overall survival among HCC patients. Infiltrative HCC is characterized by the spread of multiple minute nodules throughout the liver, without a dominant one, ultimately determining macrovascular invasion. On CT and MRI, infiltrative HCC appears as an illdefined, large mass, with variable degree of enhancement, and satellite neoplastic nodules in up to $52 \%$ of patients. On MRI, it may show restriction on diffusion weighted imaging, hyperintensity on T2- and hypointensity on T1-weighted images, and, if hepatobiliary agent is used, hypointensity on hepatobiliary phase. Infiltrative HCC must be differentiated from other liver diseases, such as focal confluent fibrosis, steatosis, amyloidosis, vascular disorders of the liver, cholangiocarcinoma, and diffuse metastatic disease. In cirrhotic patients, the identification of vascular tumor invasion of the portal vein and its differentiation from bland thrombosis is of utmost importance for patient management. On contrast enhanced CT and MRI portal vein tumor thrombosis appears as an enhancing thrombus within the portal vein, close to the main tumor and determines vein enlargement. The aim of this pictorial review is to show CT and MRI features that allow the diagnosis of infiltrative HCC and portal vein tumor thrombosis. A particular point of interest includes the tips and tricks for differential diagnosis with potential mimickers of infiltrative HCC. 
${ }_{3}^{2}$ tomography; Magnetic Resonance Imaging
4

528

6

729

8

9

10

11

12

13

14

15

16

17

18

19

20

21

22

23

24

25

26

27

28

29

30

31

32

33

34

35

36

37

38

39

40

41

42

43

44

45

46

47

48

49

50

51

52

53

54

55

56

57

58

59

60

61

62

63

64

65

Keywords: Hepatocellular carcinoma; Liver neoplasms; Differential diagnosis; Computed

政

(1)




\subsection{Introduction}

Hepatocellular carcinoma (HCC) is the fourth leading cause of cancer-related death worldwide according to the World Health Organization statistics, and it commonly arises in cirrhotic livers [1, 2]. HCC growth patterns can be categorized as nodular, massive or infiltrative at pathology $[3,4]$. Each pattern behaves differently in regard to imaging diagnosis, response to treatment, disease progression and prognosis [3-7]. Whilst the nodular type represents the most common subtype, infiltrative $\mathrm{HCC}$ is the rarest one and accounts for approximately $8-20 \%$ of all HCC cases [2-8].

Infiltrative $\mathrm{HCC}$ arises more commonly in the setting of $\mathrm{HBV}$ or $\mathrm{HBV}+\mathrm{HCV}$ co-infection and does not seem to be related to age nor duration of cirrhosis [7,9]. This infiltrative growth pattern is characterized at pathology by the spread of multiple minute nodules without a dominant one [10], which translates on cross-sectional imaging into an ill-defined permeative mass that blends into the background cirrhotic parenchyma and makes the diagnosis challenging. Infiltrative HCC has an aggressive course and overall survival of around 10 months, which is even worse in case of vascular invasion of the portal vein [513]. Infiltrative HCC may mimic other benign and malignant entities, and, until now, there are no definitive imaging criteria to clearly differentiate between $\mathrm{HCC}$ with tumor in vein from other cancers with tumor invasion. Patients with infiltrative HCC tend to have worse liver function and elevated $\alpha$-fetoprotein values $(>10,000 \mathrm{ng} / \mathrm{mL})$ with higher values as compared to patients with nodular HCC, although a-fetoprotein levels can be even normal or mildly elevated $[6,14]$.

The diagnosis of infiltrative HCC is also a therapeutic dilemma, as it narrows the therapeutic options in HCC patients $[8,11,12,15]$. The European and American Association for the Study of Liver Diseases guidelines recommend systemic treatment with sorafenib as the treatment of choice $[12,16]$ and some studies also showed controversial results on the efficacy of intra-arterial therapies when non-extensive tumor thrombosis is 
seen $[11,15]$. Due to its aggressive nature and the high likelihood of vascular invasion and extrahepatic metastatic disease, surgical resection and liver transplantation are not recommended, due to the decreased survival expectations [8, 11-13]. Transarterial radioembolization with Yttrium-90 is also considered a therapeutic option in patients with infiltrative HCC, although survival results are still controversial [17, 18]. Finn et al [19] have recently demonstrated that treatment with the combination of atezolizumab (i.e. a programmed death ligand 1 inhibitor) and bevacizumab (i.e. a monoclonal antibody targeting the vascular endothelial growth factor) results in significantly longer overall and progression-free survival as well as strikingly better patient- reported outcomes than sorafenib. Recent evidences also suggest that selected HCC patients with radiological signs of vascular invasion could be considered for transplantation, provided that they previously underwent successful treatment of the macrovascular invasion resulting in a pretransplant $\alpha$-fetoprotein $<10 \mathrm{ng} / \mathrm{ml}[20]$ and that selected patients diagnosed with HCC and macrovascular invasion without extrahepatic metastasis may have better survival if treated with resection as compared to patients treated with sorafenib [21].

Therefore, knowledge of imaging clues for diagnosis of infiltrative HCC is of utmost importance for abdominal radiologists for a proper and prompt management. The aim of this pictorial essay is to show CT and MRI features of infiltrative HCC and portal vein tumor thrombosis. A particular point of interest includes the tips and tricks for differential diagnosis with potential mimickers of infiltrative HCC.

\section{$1.2 \mathrm{CT}$ and MRI Diagnosis of infiltrative HCC}

CT and MRI have a sensitivity of $62-72 \%$ and specificity of $88-95 \%$ for the diagnosis of nodular HCC, with values depending on technique, contrast agent, and tumor size [22]. The diagnosis of nodular HCC is quite straightforward when typical imaging features (i.e. size $\geq 10 \mathrm{~mm}$, arterial phase hyperenhancement, washout on portal venous or delayed 
phase, and enhancing capsule) are detected [23]. Conversely, infiltrative HCC lacks these typical imaging features, and thus represents a diagnostic conundrum [6, 11]. In some cases, HCC is not visible and the only clue to the diagnosis may be neoplastic portal vein thrombosis [7]

CT and MRI features that suggest the diagnosis of infiltrative HCC are the presence of an ill-defined mass, usually larger than nodular HCC and without fibrotic margins, with minimal or inconsistent arterial enhancement, heterogeneous washout appearance on portal venous or delayed phases, and malignant portal vein tumor thrombosis (Figure 1) $[7,10,11,24-27]$.

The minimal or inconsistent arterial enhancement in infiltrative HCC may be due to both the infiltrative nature of the tumor and the presence of portal vein thrombosis, which results in perfusion changes that can conceal the tumor on dynamic phases; therefore, sometimes the tumor is not visible and malignant portal vein thrombosis is the only sign of infiltrative HCC $[7,10,27-30]$. CT and MRI diagnosis of malignant portal vein thrombosis in infiltrative $\mathrm{HCC}$ is based on the presence of thrombus neovascularity which corresponds to the "thread and streak sign" in angiography (i.e. a thin linear or chainlike opacification in the portal vein during early hepatic arteriography) (Figure 2) [13, 31], portal vein expansion (i.e. main portal vein diameter greater than or equal to $23 \mathrm{~mm}$ is highly specific) [32] and proximity to the main tumor or direct neoplastic portal invasion [25, 33, 34]. In case of extensive portal vein invasion, infiltrative $\mathrm{HCC}$-associated thrombosis can fill the peripheral portal vein branches, creating a dilated tumor-filled "cast" of these vessels [7].

MRI allows to appreciate the tumor as homogeneously or heterogeneously hypointense on T1-weighted images, mild to moderately hyperintense on T2-weighted images, and hyperintense, compared with surrounding liver parenchyma, on diffusion-weighted images with high $\mathrm{b}$ values $(b=500-800 \mathrm{sec} / \mathrm{mm} 2)$ due to the tightly packed cellular arrangement, with corresponding low signal on apparent diffusion coefficient (ADC) map [10, 11, 24-27, 
$30,35,36]$. The use of hepatobiliary MRI contrast agents can provide additional clues for the diagnosis of infiltrative $\mathrm{HCC}$, showing a reticular hypointense appearance on transitional and hepatobiliary phases $[10,11,30]$ and has demonstrated higher sensitivity compared to contrast enhanced CT [37].

\subsection{Mimickers of infiltrative HCC: tips and tricks for differential diagnosis}

Many benign and malignant focal lesions may mimic infiltrative HCC and tumor thrombosis on CT and MRI. The differential diagnosis is mandatory due to the different management and prognosis. Figure 3 summarize the main mimickers of infiltrative $\mathrm{HCC}$ and imaging findings that help in the differential diagnosis with infiltrative HCC. Considering that infiltrative HCC develops predominantly in cirrhotic liver, we differentiate the benign mimickers based on the presence or lack of cirrhosis. With regard to malignant mimickers, $\mathrm{HCC}$ has a higher incidence on cirrhotic liver as compared to healthy liver, whilst other malignancies usually occur in the setting of non-cirrhotic liver, but may mimic infiltrative $\mathrm{HCC}$ in some cases.

\subsection{Benign entities in cirrhotic liver}

\subsubsection{Focal Confluent Fibrosis}

Focal confluent fibrosis is a benign entity that may be identified in patients with longstanding cirrhosis [38]. On CT and MRI, both infiltrative HCC and focal confluent fibrosis show low attenuation or intensity compared to normal liver on noncontrast CT and T1weighted images, with minimal or inconsistent arterial enhancement, and mild-to-moderate hyperintensity on T2-weighted sequences [10, 26, 38]. However, focal confluent fibrosis has some distinctive imaging features that allow a confident diagnosis, including the location (typically in segments 8 and 4), the peripheral, focal, wedge-shaped appearance 
with capsular retraction, and the progressive enhancement on delayed extracellular phases (Figure 4) [26, 38]. Conversely, the acquisition of the hepatobiliary phase usually does not provide any tips for the differential diagnosis because both conditions lack the ability to uptake contrast agent $[10,26]$.

\subsubsection{Bland Thrombosis}

Cirrhotic patients may develop benign portal vein thrombosis due to portal hypertension and stasis [13, 32-34], and this bland thrombosis may be erroneously interpreted as vascular tumor invasion of the portal vein occurring in infiltrative HCC. Arterial phase plays a pivotal role for the differentiation between bland (Figure 5) and malignant (Figure 2) portal thrombosis [27]. Figure 6 summarizes the key differences between bland and malignant portal vein thrombosis. Compared to bland thrombosis, malignant tumor thrombus shows more commonly thrombus enhancement (100\% versus $8.5 \%)$, venous expansion ( $91.7 \%$ versus $10.6 \%)$, neovascularity (58.3\% versus $2.1 \%)$, and is adjacent to HCC or prior treatment site (100\% versus $21.3 \%$ ) [14]. MRI is also useful because malignant tumor thrombosis may show restricted diffusion on diffusion-weighted images and subtle T2 hyperintense signal $[7,27,30]$. In cirrhotic patients, the differential diagnosis between bland thrombosis and HCC infiltrating the portal vein has an important clinical impact because bland thrombosis may regress after anticoagulant therapy, while HCC with portal vein invasion requires systemic therapy in the form of sorafenib and thrombosis may occasionally regress after this therapy (Figure 7) [12, 16; 39].

\subsection{Benign entities in non-cirrhotic liver}

\subsubsection{Acute Hepatitis}


Acute hepatitis is a clinical diagnosis characterized by acute inflammation or injury to hepatocytes, resulting in fever, abdominal pain, jaundice, and elevation of liver biochemical lab tests. The cause of the injury may be viral (e.g. acute viral hepatitis A) or non-viral (e.g. drug-induced, alcoholic, or autoimmune acute hepatitis) [40]. Whilst normal imaging appearance of the liver does not exclude the diagnosis, CT and MRI features of acute hepatitis may include hepatomegaly, periportal edema (e.g. decreased attenuation on CT and increased T2 signal on MRI around the portal system and at the hepatic hilum), diffusely decreased parenchymal attenuation on noncontrast CT corresponding to areas of mild generalized increase in parenchymal signal intensity on T2-weighted and decreased signal intensity on T1-weighted images, and occasionally areas of steatosis on dual-phase sequence (Figure 8) [41-43]. On post-contrast CT and MRI, heterogeneous enhancement and ill-defined regions of reduced enhancement may be present with reduced uptake of hepatobiliary contrast agents and may simulate infiltrative HCC. The acute clinical onset, the presence of periportal edema, gallbladder wall thickening, and the reversibility of the heterogenous enhancement with improvement of liver function are useful tips for differential diagnosis [41-45]. Imaging findings of acute hepatitis are nonspecific and final diagnosis is clinically made.

\subsubsection{Metabolic and Storage Diseases: Steatosis, Sarcoidosis and Amyloidosis}

Metabolic and storage diseases are characterized by accumulation of storage material within the liver. Among these, steatosis, sarcoidosis and amyloidosis are benign liver conditions characterized by deposition of fat, inflammatory cells and amyloid within hepatic parenchyma, respectively.

Fat deposition with geographic distribution shows low attenuation on noncontrast CT and may have ill-defined margins, therefore potentially mimicking infiltrative HCC. However, the signal drop on opposed-phase compared to the in-phase images, the lack of mass 
effect on vessels and biliary ducts, the presence of undistorted vessels traversing through the area of focal steatosis (Figure 9), and the incidental diagnosis in otherwise healthy patients are useful tips for the differential diagnosis [10, 46].

Hepatic sarcoidosis is characterized by the presence of multiple small granulomatous lesions, scattered throughout the liver with involvement mainly of portal and periportal zones of hepatic lobules, that may lead to progressive interlobular bile duct injury with cholestasis, fibrosis of portal tracks and, rarely, evolves into cirrhosis and portal hypertension [47]. The occurrence of such multiple tiny poorly defined heterogenous nodules - most of which are less than $1 \mathrm{~cm}$ in size - may simulate the presence of an infiltrative HCC on post-contrast images (Figure 10), particularly when the liver has a cirrhotic morphology. MRI finding of hepatic sarcoidosis include the identification of granulomatous nodules as hypointense on both T1- and T2-weighted sequences [48] with hypointense periportal areas on T2-weighted images (also known as T2 halo sign) that show poor enhancement on post-contrast images [49]. In rare cases, the granulomatous lesions may coalesce and imaging demonstrates large confluent hepatic lesions [50,51]. Some of the tips that may help point towards a diagnosis of sarcoidosis include the patency of portal vein branches, the presence of concomitant splenic granulomas, and the identification of typical chest imaging features.

Hepatic amyloidosis is a rare infiltrative disease affecting the hepatic parenchyma characterized by deposition of amyloid within the space of Disse along the sinusoids or along the blood vessel walls that may lead to compression and near disappearance of hepatocytes. The infiltrative appearance of amyloidosis may simulate infiltrative HCC. CT and MRI findings of hepatic amyloidosis include hepatomegaly, heterogeneously decreased parenchymal attenuation on noncontrast $\mathrm{CT}$, and focal hypoattenuating areas on post-contrast phases (Figure 11) - probably related to impaired blood flow - that tend 

$2_{3}^{2} 1$ final diagnosis is made at pathology.

to homogenize on delayed images [52]. However, imaging findings are nonspecific and

\subsubsection{Vascular Diseases of the Liver}

Vascular diseases of the liver are rare conditions that affect the hepatic vascular system at a presinusoidal (e.g. Rendu-Osler-Weber disease, portal vein thrombosis, extrinsic compression of portal vein, aneurysmal dilatation of the portal vein, sclerosing granulomas due to schistosomiasis, sarcoidosis or tubercolosis), intrasinusoidal (i.e. chronic hepatitis, cirrhosis, peliosis hepatis, or storage of substances such as fatty liver or amyloidosis) or post-sinusoidal (e.g. Budd-Chiari syndrome, sinusoidal obstruction syndrome, right heart failure) level $[53,54]$. Regardless of the location, the obstacle in the hepatic vascular system leads to changes of hepatic parenchymal enhancement that may simulate the presence of an infiltrative HCC.

Portal vein thrombosis has been discussed above among the benign entities in cirrhotic liver. Among the other vascular diseases of the liver, Rendu-Osler-Weber disease and Budd-Chiari syndrome will be discussed below as examples.

Rendu-Osler-Weber disease, also known as hereditary hemorrhagic telangiectasia is characterized by widespread cutaneous, mucosal and visceral arteriovenous malformations that can involve lung, brain, or liver [53]. Specifically, liver vascular malformations range from tiny telangiectases to discrete arteriovenous malformations including arteriovenous, portovenous, and arterioportal shunts. Imaging features of RenduOsler-Weber disease include a transient mosaic type heterogeneous perfusion pattern of the liver in the arterial phase due to multiple arterovenous shunts and telangiectases that is no longer seen in the portal venous phase (Figure 12), hepatic artery, hepatic vein and portal vein dilatation, opacification of the hepatic veins in the arterial phase in case of hepatic arteriosystemic venous shunt and, occasionally, FNH lesions $[53,55,56]$. 
Budd-Chiari syndrome is a vascular liver disorder related to a hepatic venous outflow obstruction at the level of the hepatic veins or at the junction of the inferior vena cava and right atrium. In the acute phase of the disease, CT and MRI findings include heterogenous liver enhancement (Figure 13) characterized by decreased enhancement on arterial phase of more congested peripheral liver and increased enhancement of central portion of the liver [57]. In subacute and chronic phases, some of the CT and MR imaging features include focal nodular hyperplasia-like lesions, peripheral hepatic atrophy with compensatory hypertrophy of the central regions, and irregular hepatic contour due to fibrosis [57]. Useful tips that should prompt towards the diagnosis of Budd-Chiari syndrome include the occurrence in young women, the "nutmeg" appearance on contrast enhanced CT and MRI, the identification of occlusion of hepatic veins or inferior vena cava but patency of portal vein, the presence of intra- or extra-hepatic collateral circulation bypassing venous obstruction (which indicates a chronic process), and the splenic involvement if the etiology is a hematological disorder $[53,54,57]$.

\subsection{Malignant mimickers of infiltrative HCC}

\subsubsection{Intrahepatic Cholangiocarcinoma}

Intrahepatic cholangiocarcinoma can appear as ill-defined, large masses with low attenuation or intensity on noncontrast CT or T1-weighted images and hyperintensity on T2-weighted images [10, 58], and can mimic infiltrative HCC. Nonetheless, cholangiocarcinomas have some peculiar characteristics such as the presence of peripheral rim-like enhancement on arterial phase on both CT and MRI with gradual centripetal delayed enhancement due to central fibrotic stroma, and vascular encasement $[10,25,58]$. Moreover, the presence of dilatation of biliary ducts should favour the diagnosis of cholangiocarcinoma [10]. In case of larger lesions, cholangiocarcinoma 
causes an encasement of the vessels without definitive tumor thrombosis, a radiological sign that is helpful for differential diagnosis with HCC. However, it is important to know that, although rarely, cholangiocarcinoma with intravascular tumor thrombosis is possible (Figure 14).

\subsubsection{Lymphoma}

Hepatic lymphoma is uncommon and broadly includes secondary hepatic involvement (most common) and primary hepatic lymphoma, which is extremely rare. Hepatic lymphoma may present with multifocal lesions or diffuse infiltration or as an ill-defined mass in the porta hepatis in up to $90 \%$ of cases of secondary hepatic involvement, and in about $35 \%$ of cases of primary hepatic lymphoma (indicating a poor prognosis). This presentation of hepatic lymphoma may potentially simulate infiltrative HCC considering that both these entities may occur in patients who have cirrhosis with viral hepatitis [59, $60]$. In case of multifocal lesions, lymphomatous nodules are usually multiple with variable size and show hypoattenuation on non-contrast CT, hypointense on T1- and hyperintense on T2-weighted images and commonly enhance to a lesser degree than the liver parenchyma on all post-contrast phases (Figure 16) [60-63]. Lymphomatous infiltration of tumour cells into the portal tracts as well as sinusoids may demonstrate hepatosplenomegaly and subtle T2-hyperintensity with diffusion restriction (Figure 15), findings that can be also detected in patients with infiltrative HCC [61-64]. Some tips that favour the diagnosis of hepatic lymphoma include the normal levels of a-fetoprotein, the presence of lymphadenopathies below the level of the renal veins, and the presence of vascular encasement without thrombosis $[60,61,64,65]$.

\subsubsection{Diffuse Metastatic Disease}


6 2790 8

Hepatic metastases usually present as discrete nodules, with ring enhancement on postcontrast phases. Less commonly, liver metastases show a diffuse metastatic spread with an infiltrative appearance mimicking infiltrative HCC [66-68]. The clinical relevance of its prompt recognition is particularly related to the potential acute hepatic failure related to the diffuse intrasinusoidal spread of the hepatic metastases [66-69]. Indeed, as demonstrated at pathology, neoplastic cells diffusely infiltrate the hepatic sinusoids, invade branches of the hepatic and portal veins, and, as a consequence of pressure atrophy or vascular infarction, hepatocytes may be destroyed resulting in liver failure and a rapidly fatal course [69]. The absence of classic discrete tumor masses may lead to underestimation of the tumor burden on CT and MRI [68]. CT and MRI may reveal multiple ill-defined masses scattered throughout the liver, hypovascular on post-contrast phases and being demonstrated as diffuse small high-intensity areas on T2-weighted images and diffuse weighted images [66].

Pseudocirrhosis may occur in patients with liver metastases as a response to chemotherapy and may also lead to portal hypertension that may regress completely after chemotherapy discontinuation [70,71]. Although both pseudocirrhosis with diffuse metastases and infiltrative HCC can present with a cirrhotic-shaped liver with multiple hepatic lesions with low attenuation or intensity on noncontrast CT or T1-weighted images, hyperintense on T2-weighted images, as well as restricted diffusion on diffusion weighted imaging (Figure 16) [72], the clinical setting (e.g. presence of known primary malignancy, history of cirrhosis, elevated levels of alpha fetoprotein or other tumor markers) and the typical rim-enhancing pattern of liver metastases on post-contrast phases allow a confident diagnosis.

\subsection{Summary}


312 The diagnosis of infiltrative HCC and its eventually associated neoplastic thrombosis may 1 ${ }_{3}^{213}$ be challenging. Considering the poor prognosis of infiltrative $\mathrm{HCC}$ and the different 414
314 6 3/15 bland thrombosis, abdominal radiologists are required to know CT and MRI features of 8 13.9 11 1317 


\section{References}

1. Siegel RL, Miller KD, Jemal A. Cancer statistics, 2019. CA Cancer J Clin. 2019; 69:7-34.

2. Ferlay J, Soerjomataram I, Ervik M, Dikshit R, Eser S, Mathers C et al. GLOBOCAN 2012 v1.0, Cancer Incidence and Mortality Worldwide: IARC CancerBase No. 11. Lyon, France: International Agency for Research on Cancer; 2013.

3. Trevisani F, Caraceni $\mathrm{P}$, Bernardi M, D'Intino PE, Arienti $\mathrm{V}$, Amorati $\mathrm{P}$, et al. Gross pathologic types of hepatocellular carcinoma in Italian patients. Relationship with demographic, environmental, and clinical factors. Cancer. 1993; 72:1557-1563.

4. Kojiro M. Histopathology of liver cancers. Best Pract Res Clin Gastroenterol 2005;19:39-62.

5. He J, Shi J, Fu X, Mao L, Zhou T, Qiu Y, et al. The Clinicopathologic and Prognostic Significance of Gross Classification on Solitary Hepatocellular Carcinoma After Hepatectomy. Medicine (Baltimore). 2015 Aug;94(32):e1331. doi:

10.1097/MD.0000000000001331.

6. Yopp AC, Mokdad A, Zhu H, Mansour JC, Balch GC, Choti MA, et al. Infiltrative Hepatocellular Carcinoma: Natural History and Comparison with Multifocal, Nodular Hepatocellular Carcinoma. Ann Surg Oncol. 2015;22 Suppl 3:S1075-82. doi: 10.1245/s10434-015-4786-7.

7. Demirjian A, Peng P, Geschwind JF, Cosgrove D, Schutz J, Kamel IR, et al. Infiltrating hepatocellular carcinoma: seeing the tree through the forest. $J$ Gastrointest Surg. 2011;15(11):2089-97. doi: 10.1007/s11605-011-1614-7.

8. Dendy MS, Camacho JC, Ludwig JM, Krasinskas AM, Knechtle SJ, Kim HS. Infiltrative Hepatocellular Carcinoma With Portal Vein Tumor Thrombosis Treated With a Single High-Dose Y90 Radioembolization and Subsequent Liver 
Transplantation Without a Recurrence. Transplant Direct. 2017;3(9):e206. doi: 10.1097/TXD.0000000000000707.

9. Benvegnù L, Noventa F, Bernardinello E, Pontisso P, Gatta A, Alberti A. Evidence for an association between the aetiology of cirrhosis and pattern of hepatocellular carcinoma development. Gut. 2001;48(1):110-5. doi: 10.1136/gut.48.1.110.

10. Reynolds AR, Furlan A, Fetzer DT, Sasatomi E, Borhani AA, Heller MT, eta al. Infiltrative hepatocellular carcinoma: what radiologists need to know. Radiographics. 2015;35(2):371-86. doi: 10.1148/rg.352140114.

11. Kneuertz PJ, Demirjian A, Firoozmand A, Corona-Villalobos C, Bhagat N, Herman $J$, et al. Diffuse infiltrative hepatocellular carcinoma: assessment of presentation, treatment, and outcomes. Ann Surg Oncol. 2012; 19:2897-907. doi: 10.1245/s10434-012-2336-0..

12. EASL - European Association for the Study of the Liver, Clinical Practice Guidelines: Management of hepatocellular carcinoma. J Hepatol. 2018; 69:182-236. doi: 10.1016/j.jhep.2018.03.019. Erratum in: J Hepatol. 2019;70:817.

13. Canellas R, Mehrkhani F, Patino M, Kambadakone A, Sahani D. Characterization of Portal Vein Thrombosis (Neoplastic Versus Bland) on CT Images Using SoftwareBased Texture Analysis and Thrombus Density (Hounsfield Units). American Journal of Roentgenology. 2016; 207:W81-W87. doi:10.2214/AJR.15.15928

14. Sherman CB, Behr S, Dodge JL, Roberts JP, Yao FY, Mehta N. Distinguishing Tumor From Bland Portal Vein Thrombus in Liver Transplant Candidates With Hepatocellular Carcinoma: the A-VENA Criteria. Liver Transpl. 2019; 25:207-216. doi:10.1002/lt.25345.

15. Lopez RR Jr, Pan SH, Hoffman AL, Ramirez C, Rojter SE, Ramos H, et al. Comparison of transarterial chemoembolization in patients with unresectable, 
diffuse vs focal hepatocellular carcinoma. Arch Surg. 2002;137:653-7; discussion 657-8. doi: 10.1001/archsurg.137.6.653.

16. Heimbach JK, Kulik LM, Finn RS, Sirlin CB, Abecassis MM, Roberts LR, Zhu AX, Murad MH, Marrero JA. AASLD guidelines for the treatment of hepatocellular carcinoma. Hepatology. 2018; 67:358-380. doi: 10.1002/hep.29086.

17. Sposito C, Mazzaferro V. The SIRveNIB and SARAH trials, radioembolization vs. sorafenib in advanced HCC patients: reasons for a failure, and perspectives for the future. Hepatobiliary Surg Nutr. 2018; 7:487-489. doi:10.21037/hbsn.2018.10.06

18. Spreafico C, Sposito C, Vaiani M, Cascella T, Bhoori S, Morosi C, et al. Development of a prognostic score to predict response to Yttrium-90 radioembolization for hepatocellular carcinoma with portal vein invasion. J Hepatol. 2018; 68:724-732. doi: 10.1016/j.jhep.2017.12.026.

19. Finn RS, Qin S, Ikeda M, Galle PR, Ducreux M, Kim TY, et al. Atezolizumab plus Bevacizumab in Unresectable Hepatocellular Carcinoma. N Engl J Med. 2020; 382:1894-1905. doi: 10.1056/NEJMoa1915745.

20. Assalino M, Terraz S, Grat M, Lai Q, Vachharajani N, Gringeri E, et al. Liver transplantation for hepatocellular carcinoma after successful treatment of macrovascular invasion - a multi-center retrospective cohort study. Transpl Int. 2020; 33:567-575. doi: 10.1111/tri.13586.

21. Mei J, Li SH, Wang QX, Lu LH, Ling YH, Zou JW, et al. Resection vs. Sorafenib for Hepatocellular Carcinoma With Macroscopic Vascular Invasion: A Real World, Propensity Score Matched Analytic Study. Front Oncol. 2020;10:573. doi: 10.3389/fonc. 2020.00573 .

22. Lee S, Kim SS, Roh YH, Choi JY, Park MS, Kim MJ. Diagnostic Performance of CT/MRI Liver Imaging Reporting and Data System v2017 for Hepatocellular 
Carcinoma: A Systematic Review and Meta-Analysis. Liver Int. 2020; 40:14881497.

23. ACR CEUS LI-RADS® V2017. (last access on 20/04/2020)

24. Kanematsu M, Semelka RC, Leonardou P, Mastropasqua M, Lee JK. Hepatocellular carcinoma of diffuse type: MR imaging findings and clinical manifestations. J Magn Reson Imaging. 2003; 18:189-95. doi: 10.1002/jmri.10336.

25. Kim YK, Han YM, Kim CS. Comparison of diffuse hepatocellular carcinoma and intrahepatic cholangiocarcinoma using sequentially acquired gadolinium-enhanced and Resovist-enhanced MRI. Eur J Radiol. 2009; 70:94-100.

26. Park YS, Lee CH, Kim BH, Lee J, Choi JW, Kim KA, et al. Using Gd-EOB-DTPAenhanced 3-T MRI for the differentiation of infiltrative hepatocellular carcinoma and focal confluent fibrosis in liver cirrhosis. Magn Reson Imaging. 2013; 31:1137-42. doi: 10.1016/j.mri.2013.01.011.

27. Lim S, Kim YK, Park HJ, Lee WJ, Choi D, Park MJ. Infiltrative hepatocellular carcinoma on gadoxetic acid-enhanced and diffusion-weighted MRI at 3.0T. J Magn Reson Imaging. 2014; 39:1238-45. doi: 10.1002/jmri.24265.

28. Thian YL, Low AS, Chow PK, Ooi LL, Chung AY, Low SC, Xie W, Thng CH. Atypical enhancement pattern of hepatocellular carcinoma with portal vein thrombosis on multiphasic CT. Ann Acad Med Singapore. 2011;40:454-9.

29. Danila M, Sporea I, Popescu A, Șirli R. Portal vein thrombosis in liver cirrhosis - the added value of contrast enhanced ultrasonography. Med Ultrason. 2016;18:218-33. doi: 10.11152/mu.2013.2066.182.pvt.

30. Rosenkrantz AB, Lee L, Matza BW, Kim S. Infiltrative hepatocellular carcinoma: comparison of MRI sequences for lesion conspicuity. Clin Radiol. 2012; 67:e105-11. doi: 10.1016/j.crad.2012.08.019. 
31. Raab BW. The thread and streak sign. Radiology. 2005; 236:284-5. doi:10.1148/radiol.2361030114

32. Tublin ME, Dodd GD 3rd, Baron RL. Benign and malignant portal vein thrombosis: differentiation by CT characteristics. AJR Am J Roentgenol. 1997; 168:719-23. doi:10.2214/ajr.168.3.9057522

33. Piscaglia F, Gianstefani A, Ravaioli M, Golfieri R, Cappelli A, Giampalma E, et al. Criteria for diagnosing benign portal vein thrombosis in the assessment of patients with cirrhosis and hepatocellular carcinoma for liver transplantation. Liver Transpl. 2010; 16:658-67. doi: 10.1002/lt.22044.

34. Margini C, Berzigotti A. Portal vein thrombosis: The role of imaging in the clinical setting. Dig Liver Dis. 2017;49(2):113-20. doi:10.1016/j.dld.2016.11.013

35. Choi JY, Lee JM, Sirlin CB. CT and MR imaging diagnosis and staging of hepatocellular carcinoma: part II. Extracellular agents, hepatobiliary agents, and ancillary imaging features. Radiology. 2014;273:30-50. doi:

10.1148/radiol.14132362.

36. Yu NC, Chaudhari V, Raman SS, Lassman C, Tong MJ, Busuttil RW, et al. CT and MRI improve detection of hepatocellular carcinoma, compared with ultrasound alone, in patients with cirrhosis. Clin Gastroenterol Hepatol. 2011; 9:161-7. doi: 10.1016/j.cgh.2010.09.017.

37. Bae JS, Lee JM, Yoon JH, Jang S, Chung JW, Lee KB, et al. How to Best Detect Portal Vein Tumor Thrombosis in Patients with Hepatocellular Carcinoma Meeting the Milan Criteria: Gadoxetic Acid-Enhanced MRI versus Contrast-Enhanced CT. Liver Cancer. 2020; 9:293-307. doi: 10.1159/000505191.

38. Brancatelli G, Baron RL, Federle MP, Sparacia G, Pealer K. Focal confluent fibrosis in cirrhotic liver: natural history studied with serial CT. AJR Am J Roentgenol. 2009; 192:1341-7. doi: 10.2214/AJR.07.2782. 
39. Bruix J, Sherman M; American Association for the Study of Liver Diseases. Management of hepatocellular carcinoma: an update. Hepatology. 2011; 53:1020-2. doi: 10.1002/hep.24199.

40. Ryder SD, Beckingham IJ. ABC of diseases of liver, pancreas, and biliary system: Acute hepatitis. BMJ. 2001; 322:151-3. doi: 10.1136/bmj.322.7279.151.

41. Park SJ, Kim JD, Seo YS, Park BJ, Kim MJ, Um SH, et al. Computed tomography findings for predicting severe acute hepatitis with prolonged cholestasis. World $\mathrm{J}$ Gastroenterol. 2013; 19:2543-9. doi: 10.3748/wjg.v19.i16.2543.

42. Murakami T, Baron RL, Peterson MS. Liver necrosis and regeneration after fulminant hepatitis: pathologic correlation with CT and MR findings. Radiology. 1996; 198:239-242. doi:10.1148/radiology.198.1.8539386

43. Onishi H, Theisen D, Zachoval R, Reiser MF, Zech CJ. Intrahepatic diffuse periportal enhancement patterns on hepatobiliary phase gadoxetate disodiumenhanced liver MR images: Do they correspond to periportal hyperintense patterns on T2-weighted images? Medicine (Baltimore). 2019; 98:e14784. doi: 10.1097/MD.0000000000014784.

44. Martin DR, Seibert D, Yang M, Salman K, Frick MP. Reversible heterogeneous arterial phase liver perfusion associated with transient acute hepatitis: findings on gadolinium-enhanced MRI. J Magn Reson Imaging. 2004; 20:838-842. doi:10.1002/jmri.20192

45. Marzola P, Maggioni F, Vicinanza E, Daprà M, Cavagna FM. Evaluation of the hepatocyte-specific contrast agent gadobenate dimeglumine for MR imaging of acute hepatitis in a rat model. J Magn Reson Imaging. 1997; 7:147-152. doi:10.1002/jmri.1880070121. 
46. Dioguardi Burgio M, Bruno O, Agnello F, Torrisi C, Vernuccio F, Cabibbo G, et al. The cheating liver: imaging of focal steatosis and fatty sparing. Expert Rev Gastroenterol Hepatol. 2016; 10:671-8. doi: 10.1586/17474124.2016.1169919.

47. Karaosmanoğlu AD, Onur MR, Saini S, Taberi A, Karcaaltincaba M. Imaging of hepatobiliary involvement in sarcoidosis. Abdom Imaging. 2015; 40:3330-7. doi: 10.1007/s00261-015-0533-6.

48. Ganeshan D, Menias CO, Lubner MG, Pickhardt PJ, Sandrasegaran K, Bhalla S. Sarcoidosis from Head to Toe: What the Radiologist Needs to Know. Radiographics. 2018; 38:1180-200. doi: 10.1148/rg.2018170157.

49. Jung G, Brill N, Poll LW, Koch JA, Wettstein M. MRI of hepatic sarcoidosis: large confluent lesions mimicking malignancy. AJR Am J Roentgenol. 2004; 183:171-3. doi: 10.2214/ajr.183.1.1830171.

50. Masuda K, Takenaga S, Morikawa K, Kano A, Ojiri H. Hepatic sarcoidosis with atypical radiological manifestations: A case report. Radiol Case Rep. 2018; 13:936939. doi: 10.1016/j.radcr.2018.06.013..

51. Kim SH, Han JK, Lee KH, Won HJ, Kim KW, Kim JS, et al. Abdominal amyloidosis: spectrum of radiological findings. Clin Radiol. 2003; 58:610-20. doi: 10.1016/s00099260(03)00142-9.

52. DeLeve LD, Valla DC, Garcia-Tsao G; American Association for the Study Liver Diseases. Vascular disorders of the liver. Hepatology. 2009; 49:1729-64. doi: 10.1002/hep.22772.

53. Valla DC. Budd-Chiari syndrome/hepatic venous outflow tract obstruction. Hepatol Int. 2018; 12:168-180. doi: 10.1007/s12072-017-9810-5.

54. Buscarini E, Gandolfi S, Alicante S, Londoni C, Manfredi G. Liver involvement in hereditary hemorrhagic telangiectasia. Abdom Radiol (NY). 2018; 43:1920-1930. doi: 10.1007/s00261-018-1671-4. 
55. Wu JS, Saluja S, Garcia-Tsao G, Chong A, Henderson KJ, White RI Jr. Liver involvement in hereditary hemorrhagic telangiectasia: CT and clinical findings do not correlate in symptomatic patients. AJR Am J Roentgenol. 2006; 187:W399W405. doi:10.2214/AJR.05.1068

56. Brancatelli G, Vilgrain V, Federle MP, Hakime A, Lagalla R, lannaccone R, et al. Budd-Chiari syndrome: spectrum of imaging findings. AJR Am J Roentgenol. 2007; 188:W168-76. doi: 10.2214/AJR.05.0168.

57. Chung YE, Kim MJ, Park YN, Choi JY, Pyo JY, Kim YC, et al. Varying appearances of cholangiocarcinoma: radiologic-pathologic correlation. Radiographics. 2009; 29:683-700. doi: 10.1148/rg.293085729.

58. Schöllkopf C, Smedby KE, Hjalgrim H, Rostgaard K, Panum I, Vinner L, et al. Hepatitis C infection and risk of malignant lymphoma. Int J Cancer. 2008; 122:188590. doi: 10.1002/ijc.23416.

59. Tomasian A, Sandrasegaran K, Elsayes KM, Shanbhogue A, Shaaban A, Menias $\mathrm{CO}$. Hematologic malignancies of the liver: spectrum of disease. Radiographics. 2015; 35:71-86. doi:10.1148/rg.351130008

60. Rajesh S, Bansal K, Sureka B, Patidar Y, Bihari C, Arora A. The imaging conundrum of hepatic lymphoma revisited. Insights Imaging. 2015; 6:679-692. doi:10.1007/s13244-015-0437-6

61. Weissleder R, Stark DD, Elizondo G, et al. MRI of hepatic lymphoma. Magn Reson Imaging. 1988; 6:675-681. doi:10.1016/0730-725x(88)90092-6

62. Colagrande S, Calistri L, Grazzini G, Nardi C, Busoni S, Morana G, et al. MRI features of primary hepatic lymphoma. Abdom Radiol (NY). 2018; 43:2277-2287. doi: 10.1007/s00261-018-1476-5. 
63. Ippolito D, Porta M, Maino C, Pecorelli A, Ragusi M, Giandola T, et al. Diagnostic approach in hepatic lymphoma: radiological imaging findings and literature review. $\mathrm{J}$ Cancer Res Clin Oncol. 2020; 146:1545-1558. doi: 10.1007/s00432-020-03205-x.

64. Semelka RC, Nimojan N, Chandana S, Ramalho M, Palmer SL, DeMulder D, et al. MRI features of primary rare malignancies of the liver: A report from four university centres. Eur Radiol. 2018; 28:1529-1539. doi: 10.1007/s00330-017-5102-7.

65. Sato K, Takeyama Y, Tanaka T, Fukui Y, Gonda H, Suzuki R. Fulminant hepatic failure and hepatomegaly caused by diffuse liver metastases from small cell lung carcinoma: 2 autopsy cases. Respir Investig. 2013; 51:98-102. doi: 10.1016/j.resinv.2012.12.004.

66. Tanaka K, Tomita H, Hisamatsu K, Hatano Y, Yoshida K, Hara A. Acute Liver Failure Associated with Diffuse Hepatic Infiltration of Malignant Melanoma of Unknown Primary Origin. Intern Med. 2015; 54:1361-1364. doi:10.2169/internalmedicine.54.3428

67. Hanamornroongruang S, Sangchay N. Acute liver failure associated with diffuse liver infiltration by metastatic breast carcinoma: A case report. Oncol Lett. 2013; 5:1250-1252. doi:10.3892/ol.2013.1165

68. Allison KH, Fligner CL, Parks WT. Radiographically occult, diffuse intrasinusoidal hepatic metastases from primary breast carcinomas: a clinicopathologic study of 3 autopsy cases. Arch Pathol Lab Med. 2004; 128:1418-1423. doi:10.1043/15432165(2004)128<1418:RODIHM>2.0.CO;2

69. Vernuccio F, Dioguardi Burgio M, Barbiera F, Cusmà S, Badalamenti G, Midiri M, et al. CT and MR imaging of chemotherapy-induced hepatopathy. Abdom Radiol (NY). 2019; 44:3312-3324. doi: 10.1007/s00261-019-02193-y.

70. Vilgrain V., Lagadec M., Ronot M., Pitfalls in Liver Imaging. Radiology 2016; 278; 1:34-51. doi:10.1148/radiol.2015142576 
71. Kang SP, Taddei T, McLennan B, Lacy J. Pseudocirrhosis in a pancreatic cancer patient with liver metastases: A case report of complete resolution of pseudocirrhosis with an early recognition and management. World J Gastroenterol 2008; 14:1622-1624. doi:10.3748/wjg.14.1622 


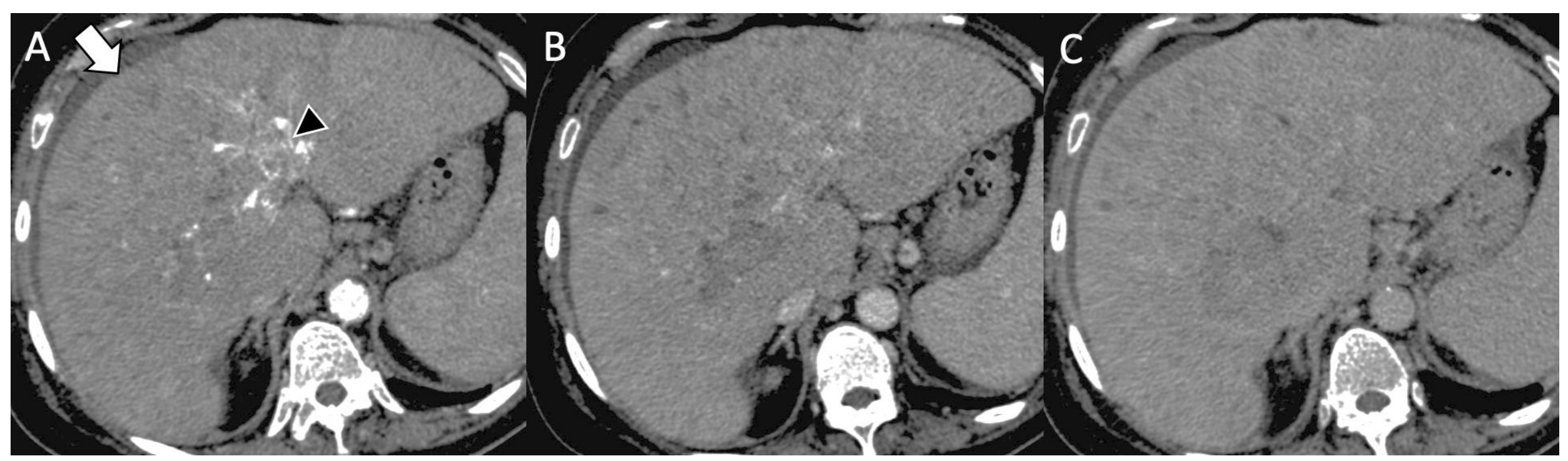

Fig. 1 - 66-year-old cirrhotic woman with infiltrative HCC. Axial CT images on (a) arterial, (b) portal venous and (c) delayed phases demonstrate a large ill-defined mass (arrow) with no arterial hyperenhancement, mild washout on portal venous and delayed phases and an hyperenhancing portal vein tumor thrombus (arrowhead) with washout on portal venous phase. The tumor itself is not clearly visible due to inconsistent enhancement on arterial phase and only minimal washout on later phases. 


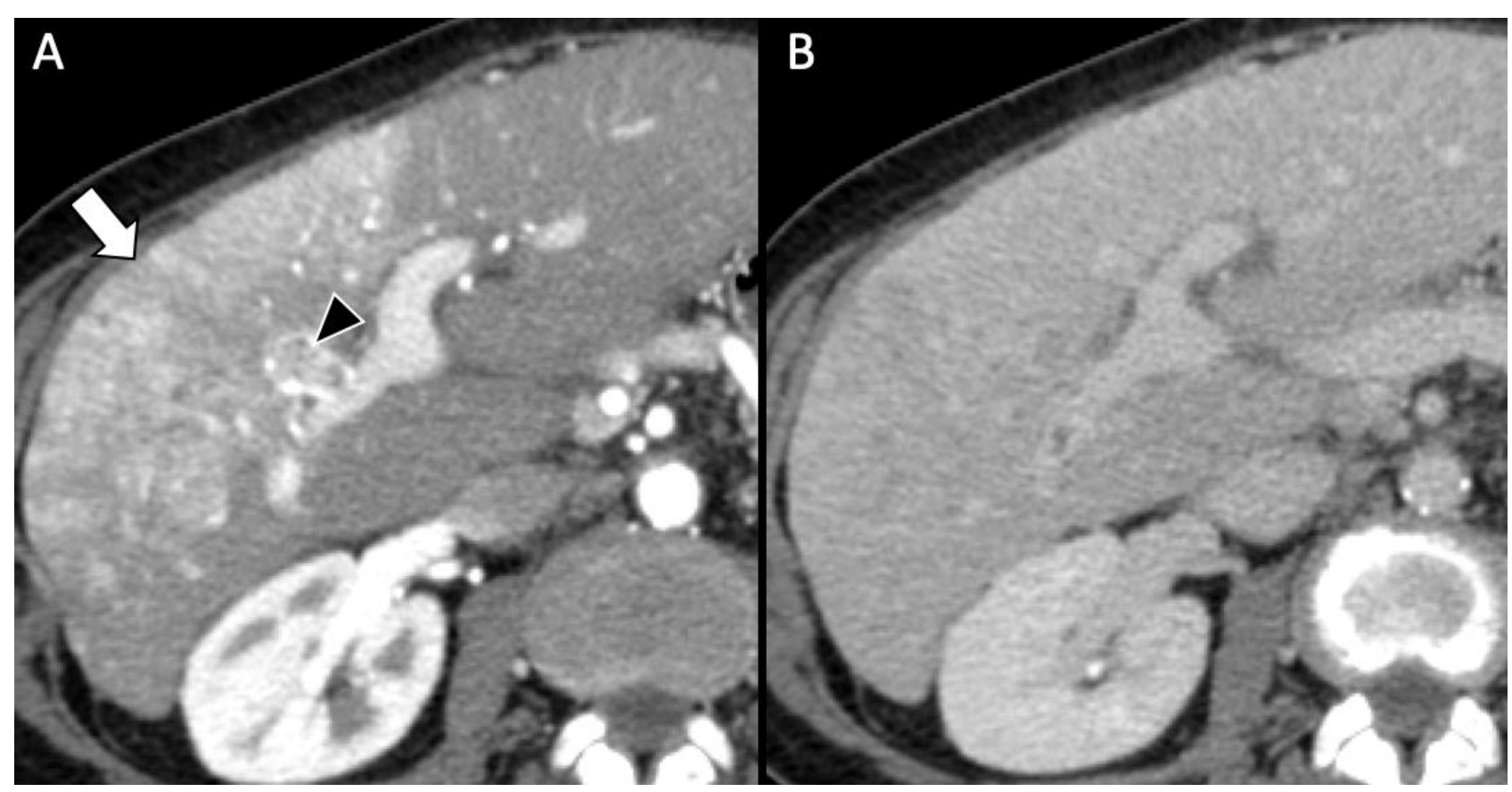

Fig. 2 - 67-year-old woman with infiltrative HCC and neoplastic tumor thrombus. Axial CT image (a) on arterial phase demonstrates an enhancing tumor thrombus (arrowhead) in the right anterior portal vein branch with the "thread and streak sign"; the malignant portal vein thrombus causes expansion of the involved portal vein branch and is adjacent to an ill-defined enhancing tumor mass (arrow) (a). Notably, the marked enhancement on arterial phase is due to both $\mathrm{HCC}$ and perfusion abnormalities related to portal vein thrombosis, thus overestimating the entity of the tumor mass. (b) On portal venous phase only some areas demonstrate washout within the ill-defined enhancing tumor mass. 
- Ill-defined mass

- Satellite nodules

- Tumor thrombus

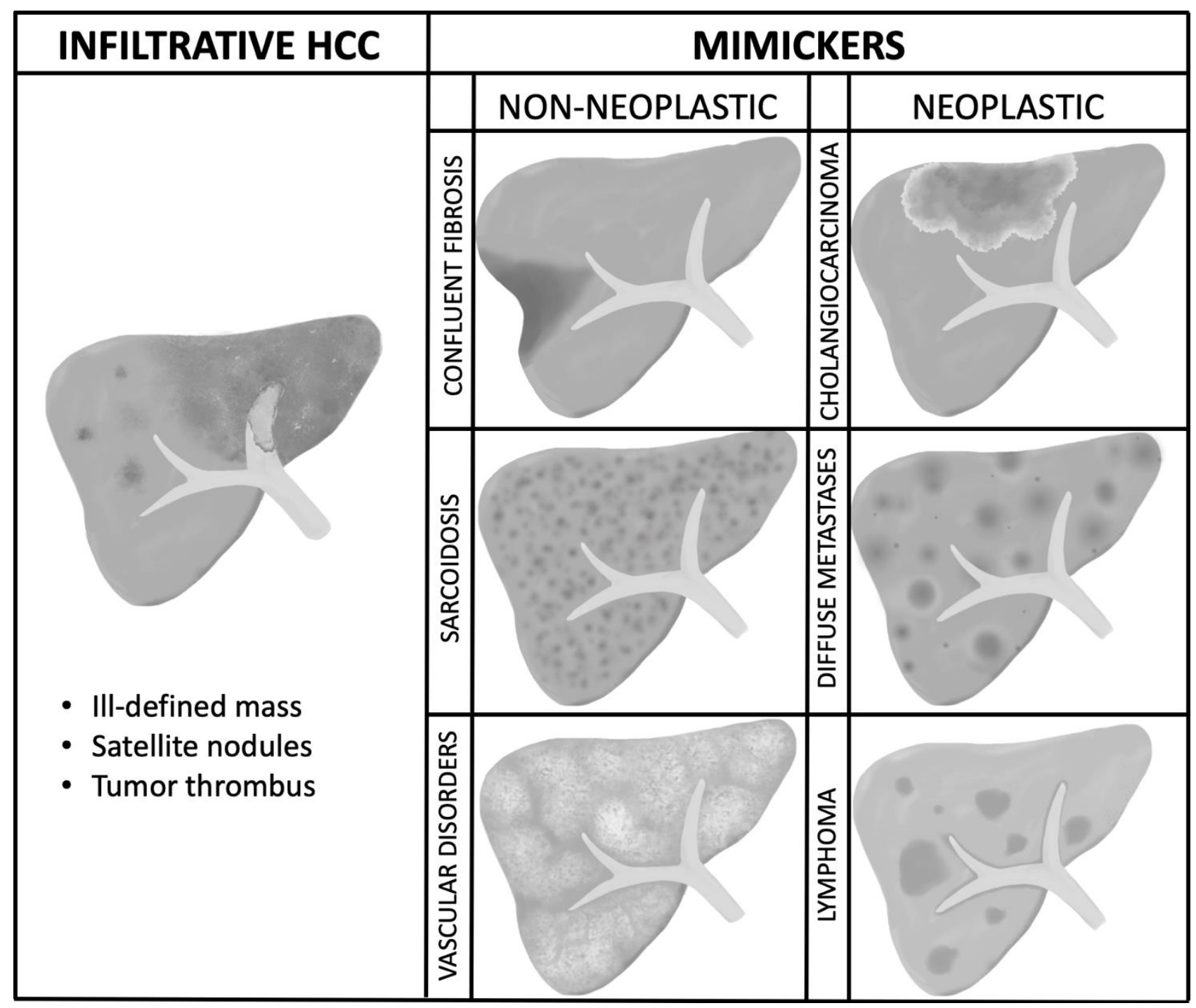

Fig. 3 - Schematic representation of main imaging features of infiltrative HCC and its benign and malignant mimickers. 


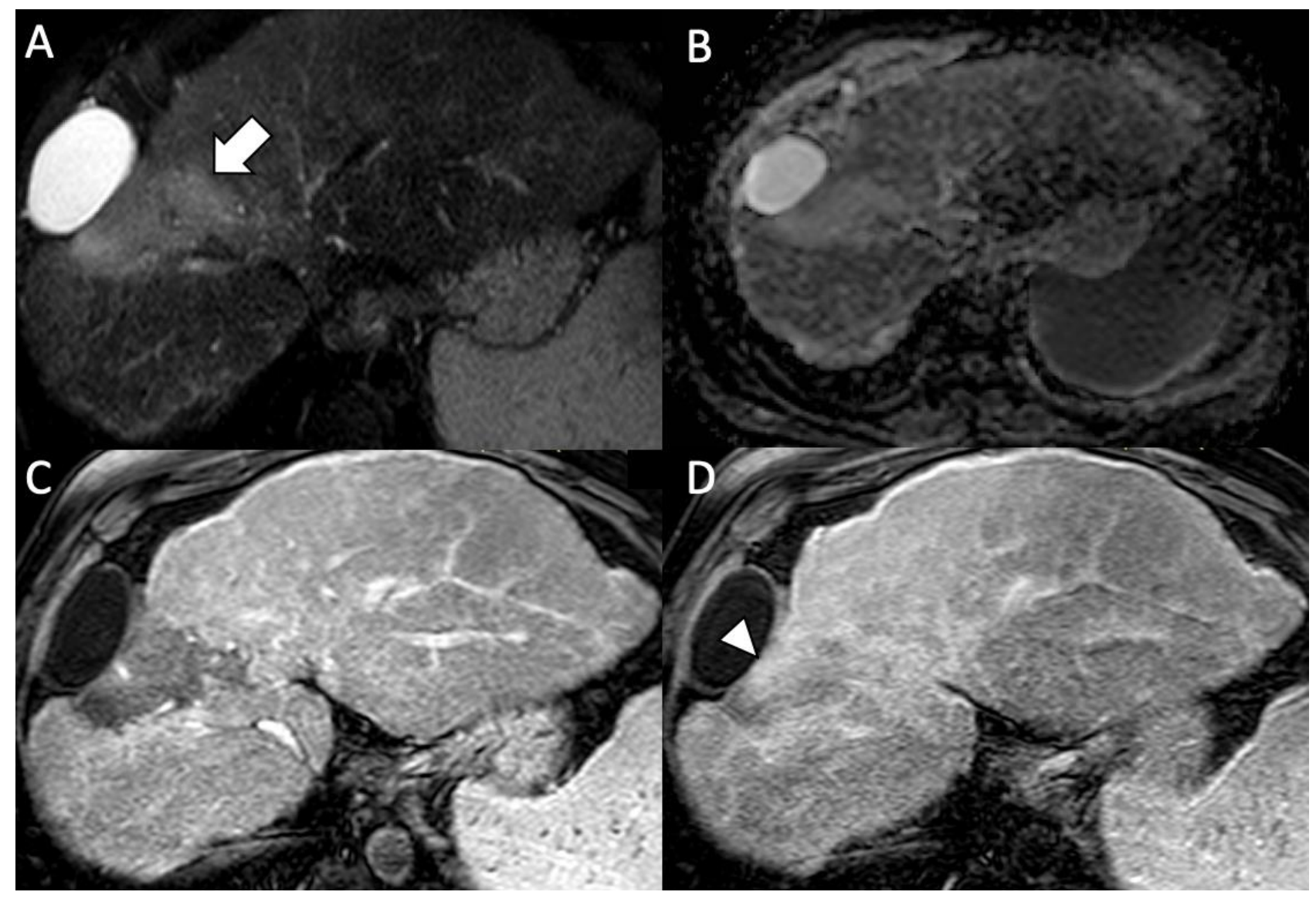

Fig. 4 - 52-year-old cirrhotic man with focal confluent fibrosis. Gadoterate meglumine MRI demonstrates on (a) T2-weighted sequence a wedge-shaped hyperintense observation (arrow) in segment VIII (b) with lack of diffusion restriction on apparent diffusion coefficient map, (c) lack of enhancement on arterial phase and (d) progressive enhancement (arrowhead) at 5-minute delayed phase. 


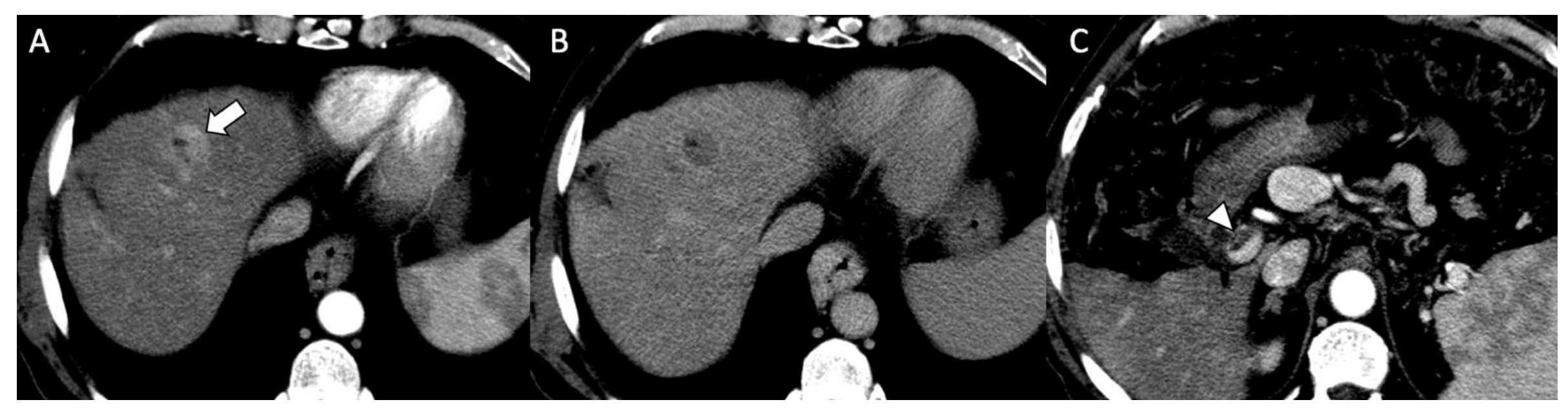

Fig. 5 - 65-year-old cirrhotic man with hepatocellular carcinoma and bland thrombus. Contrast enhanced CT on (a) arterial phase shows a $2.5 \mathrm{~cm}$ observation (arrow) with nonrim arterial phase hyperenhancement and (b) portal venous washout, consistent with hepatocellular carcinoma. In the same patient there is also (c) a portal vein thrombus (arrowhead) that lacks any contrast enhancement on arterial phase, does not show portal vein expansion, and is distant from hepatocellular carcinoma. 


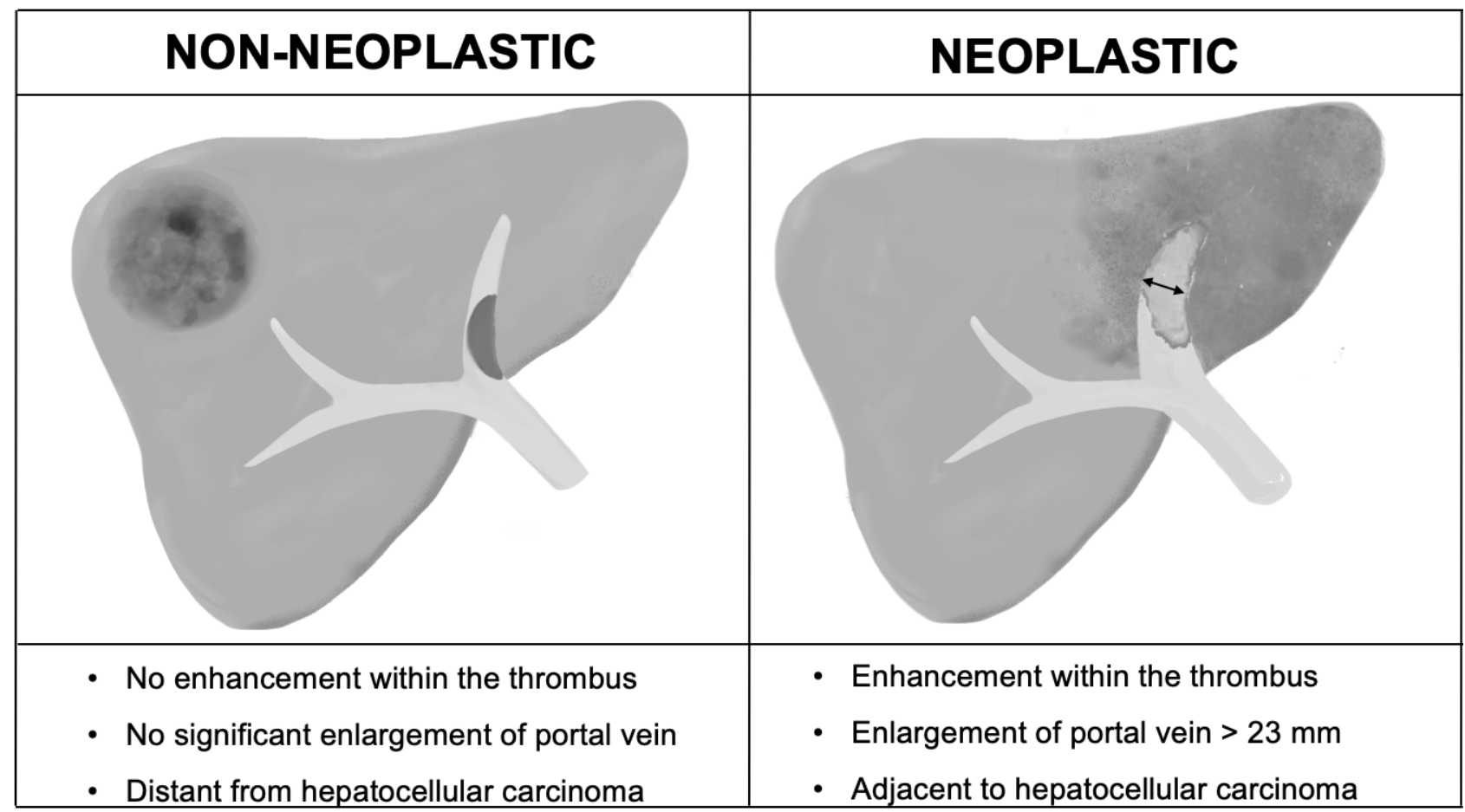

Fig. 6 - Schematic representation of main imaging features that allow the differentiation between bland and tumor thrombosis in patients with hepatocellular carcinoma. 

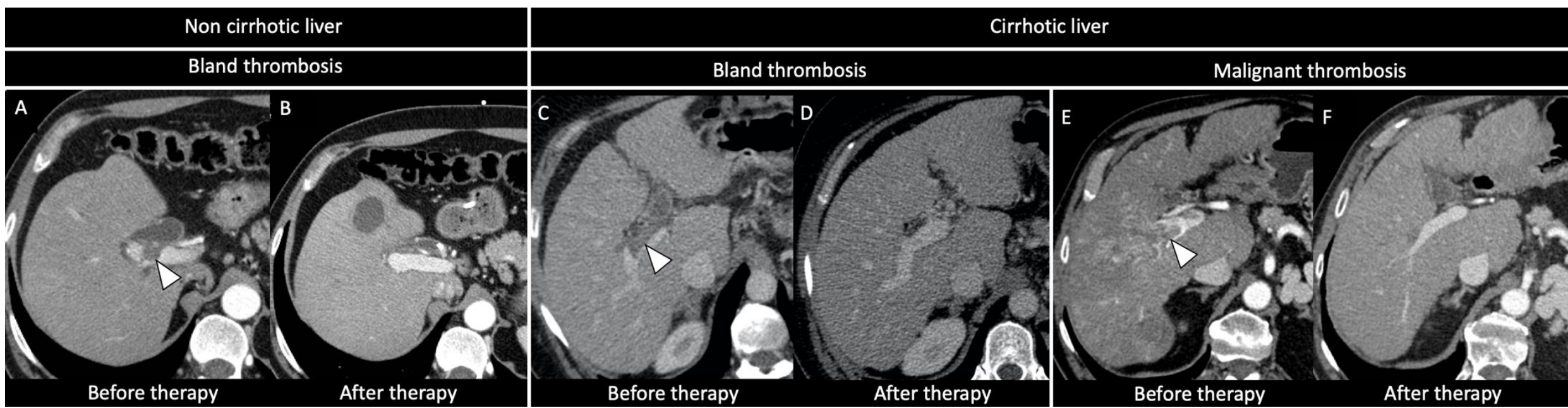

Fig. 7 - a and b: 45-year-old male-to-female transgender, in estrogenic hormone therapy with cholecystitis and bland thrombus. (a) Contrast enhanced CT on arterial phase shows a bland thrombus (arrow) in the portal vein that (b) completely regressed after anticoagulant therapy; of note, patient developed a liver abscess in segment 4 in the interval time. c and d: 56-year-old cirrhotic man with bland thrombus. (c) Contrast enhanced CT on portal venous phase shows a bland thrombus (arrow) in the portal vein that (d) completely regressed after anticoagulant therapy. e and f: 72-year-old cirrhotic man with infiltrative HCC. (e) Contrast enhanced CT on arterial phase shows an infiltrative HCC with portal vein tumor invasion (arrow) that (f) regressed after therapy with sorafenib. 


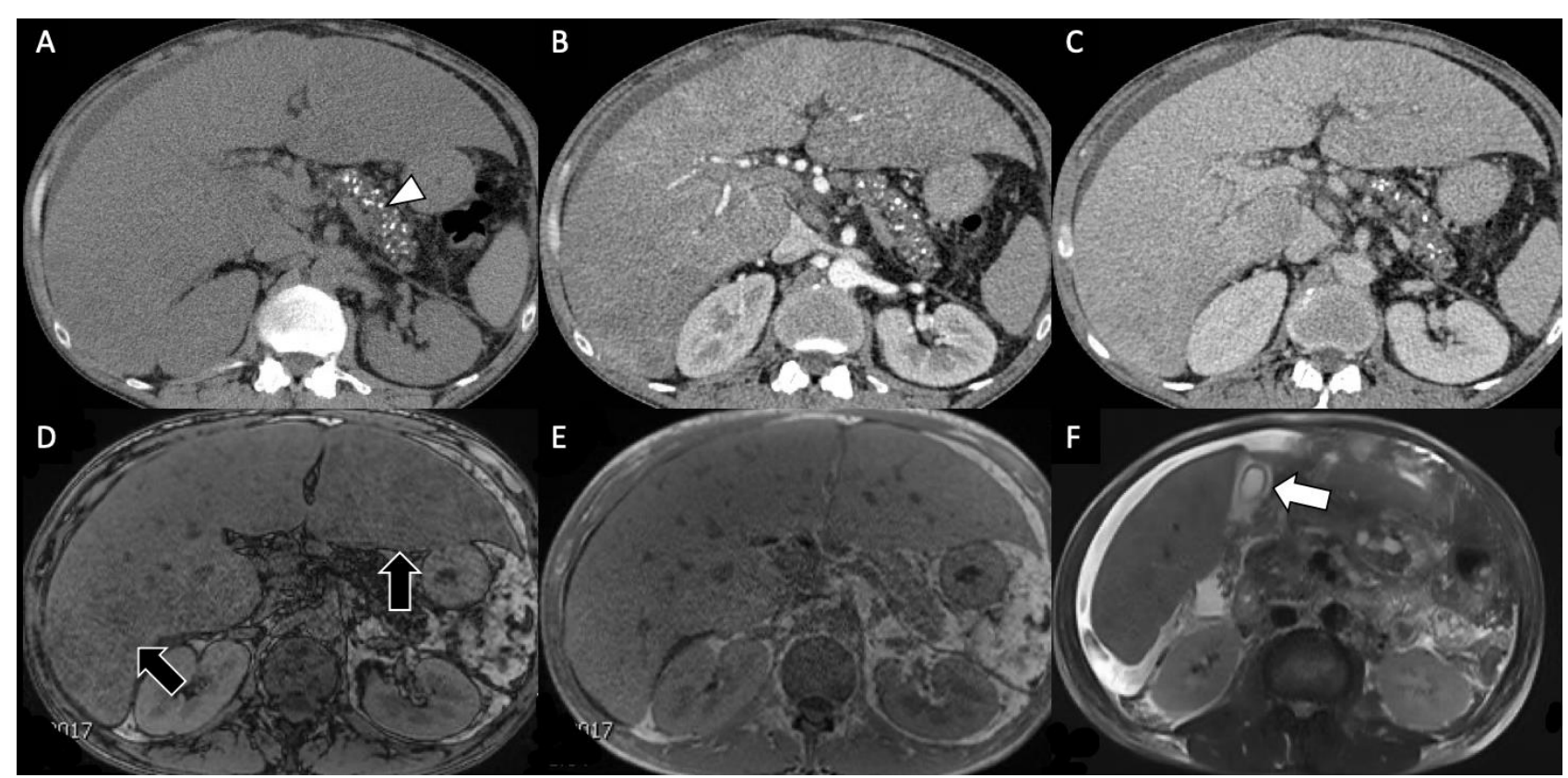

Fig. 8 - 36-year-old man with decompensated alcoholic hepatopathy and increase of cholestatic laboratory values due to acute hepatitis. Axial CT images on (a) precontrast, (b) arterial, and (c) portal venous phases show hepatomegaly with diffuse spontaneous hepatic hypoattenuation and heterogenous enhancement with ill-defined hypoattenuating areas; pancreatic calcifications (arrowhead) are also demonstrated. On MRI, dual phase sequence shows (d) signal drop (arrows) in the opposed phase compared to (e) the inphase image. (f) Ascites and gallbladder edematous wall thickening (arrow) are demonstrated on T2-weighted image. Case courtesy of blinded to reviewers. 


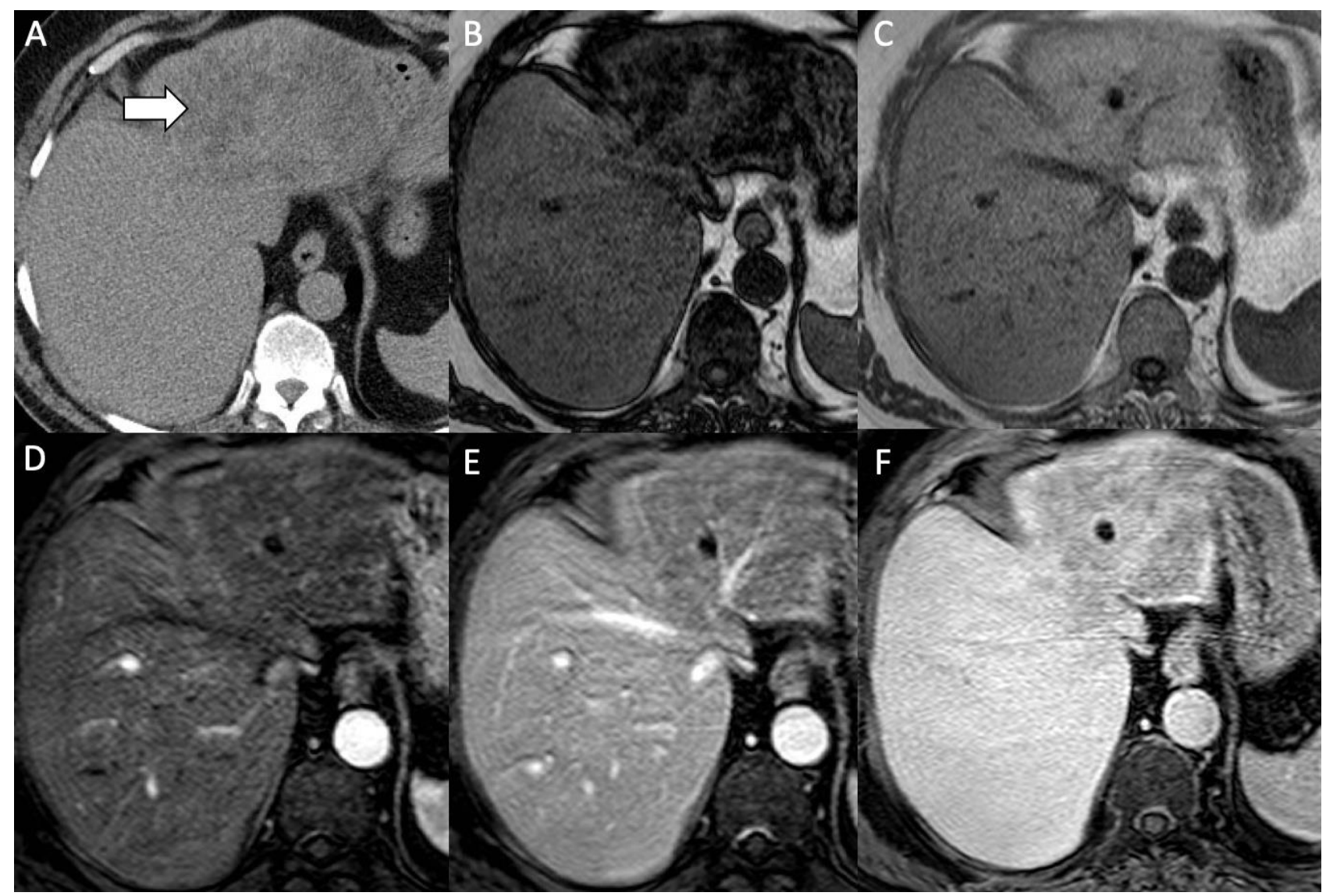

Fig. 9 - 67-year-old man with diabetes in treatment with insulin and geographical steatosis. (a) Axial CT unenhanced image showed an ill-defined hypoattenuating area (arrow) in the left lobe. Gadobenate dimeglumine-enhanced MRI demonstrated (b) marked signal drop in the opposed phase compared to (c) in-phase image, with (d) lack of enhancement on arterial phase or (e) portal venous phase with presence of undisturbed left hepatic vein and its branches traversing through the area of steatosis; (f) this area is also hypoattenuating at two hours after contrast injection; of note, in this acquisition at two hours after contrast injection, intrahepatic vessels are isointense to liver parenchyma probably due to impaired liver function and, therefore, this is similar to a transitional phase rather than an hepatobiliary phase. 

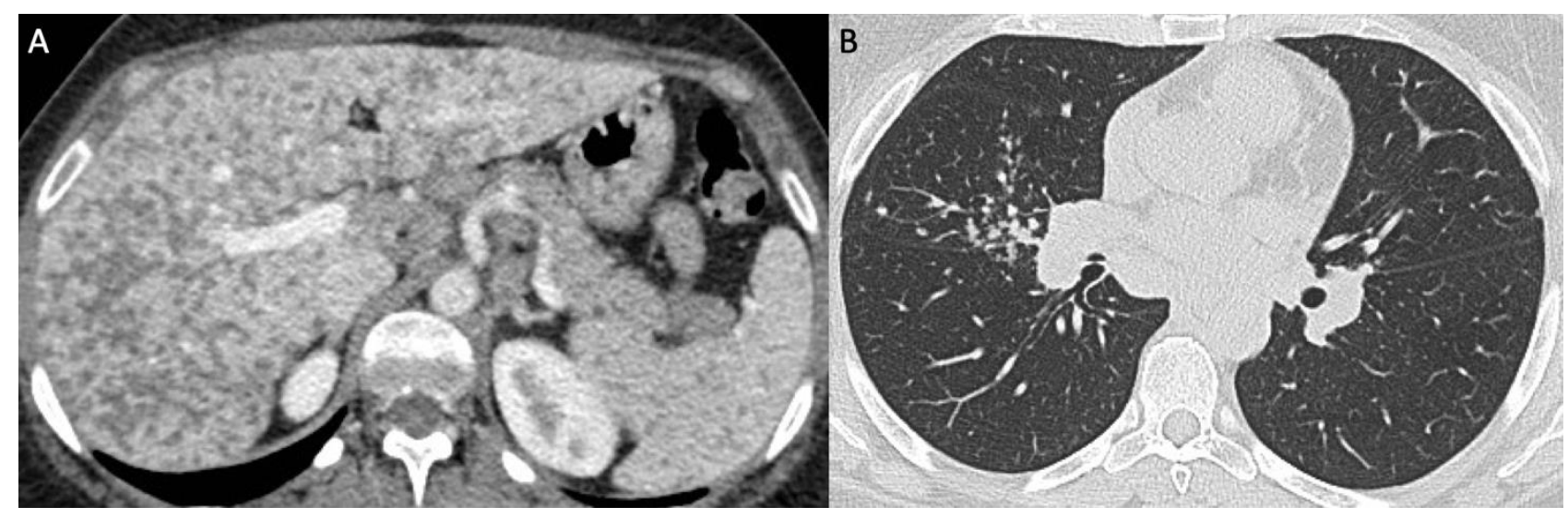

Fig. 10 - 48-year-old woman with sarcoidosis. (a) Axial CT image on portal venous phase demonstrates multiple tiny poorly defined hypoattenuating nodules scattered throughout liver and spleen. (b) Axial chest CT image in the same patient demonstrate small tiny nodules in a perilymphatic distribution along bronchovascular bundle in the medial lobe. 


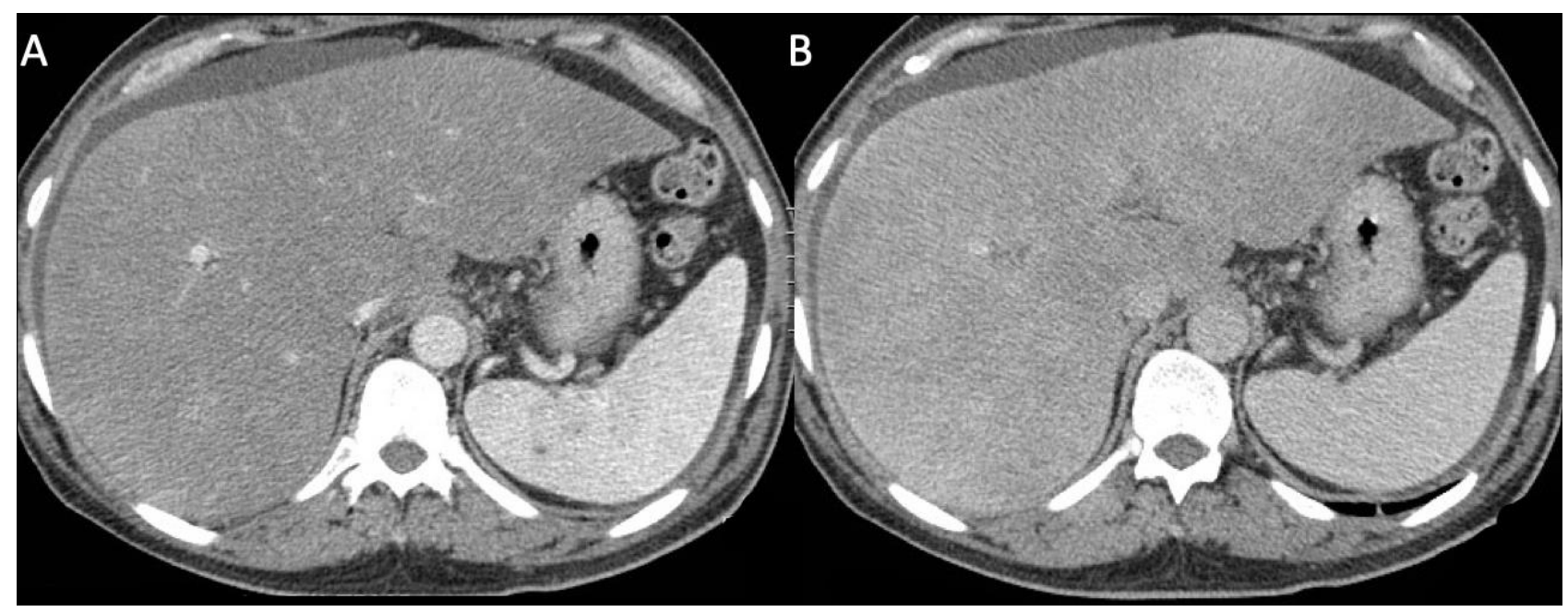

Fig. 11 - 56-year-old man with amyloidosis; the patient was admitted for abdominal pain with weight loss and asthenia for three months. Axial CT images on (a) arterial and (b) portal venous phases demonstrate hepatomegaly, heterogeneously decreased parenchymal attenuation on arterial phase and heterogeneous contrast enhancement with diffuse low density areas scattered throughout liver parenchyma on portal venous phase. These CT findings suggested diffuse infiltrative disease, so liver biopsy was performed. The histological examination showed perisinusoidal deposits of an amorphous eosinophilic material stained by Congo red stain. Biopsy results led to a final diagnosis of diffuse amyloidosis. 


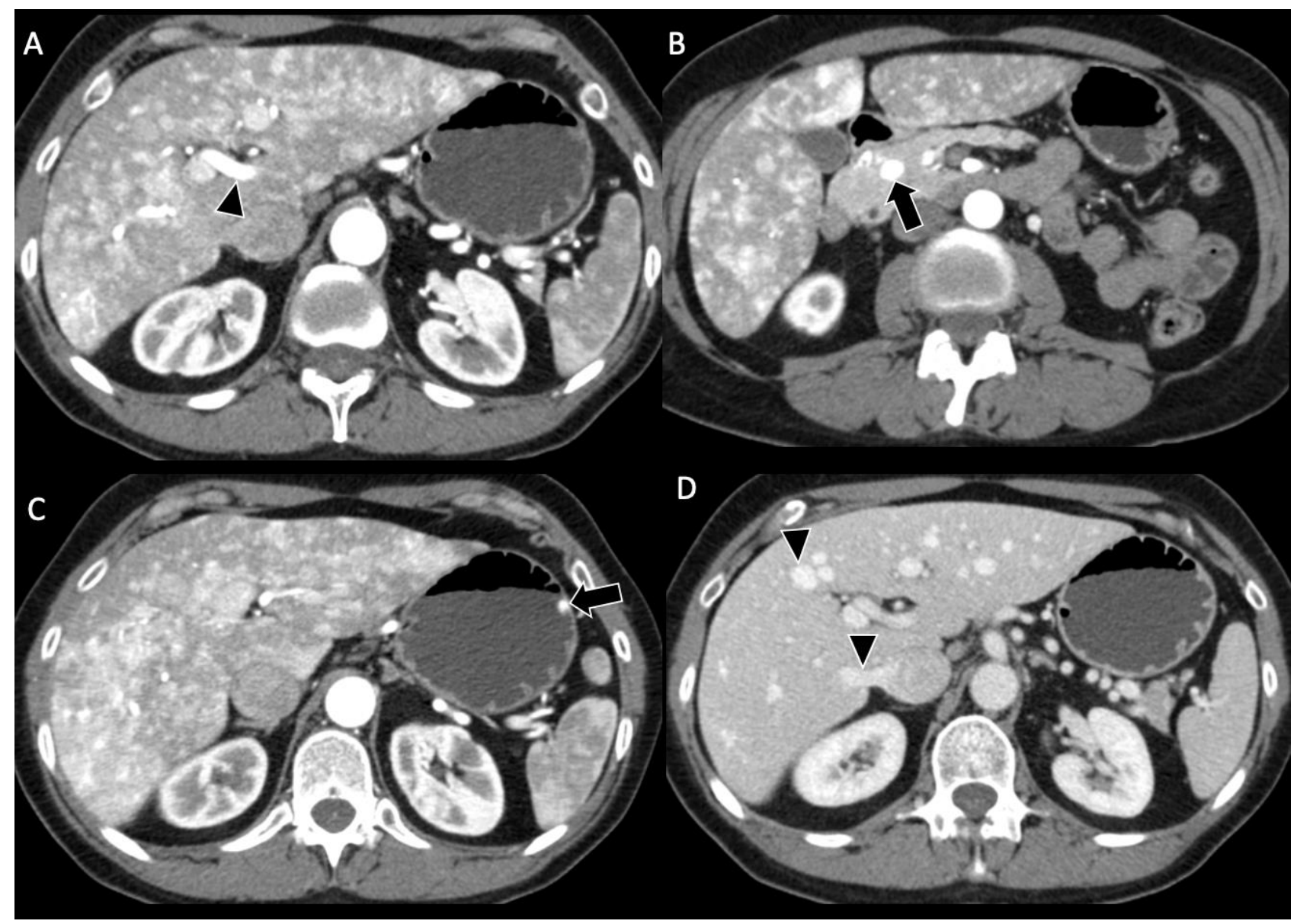

Fig. 12 - 55-year-old woman with Rendu-Osler-Weber disease; the patient was

hospitalized several times for hematemesis. (a, b and c) Axial CT images on arterial phase at three different levels show a mosaic type heterogeneous perfusion pattern of the liver due to numerous arterio-portal and arterio-venous shunts, enlargement of hepatic artery (arrowhead in a), and arterio-venous shunts in the pancreatic head (arrow in b) and in the gastric wall (arrow in c). (d) Portal venous phase image demonstrates normal attenuation of the liver parenchyma and enlargement of hepatic veins (arrowheads). 


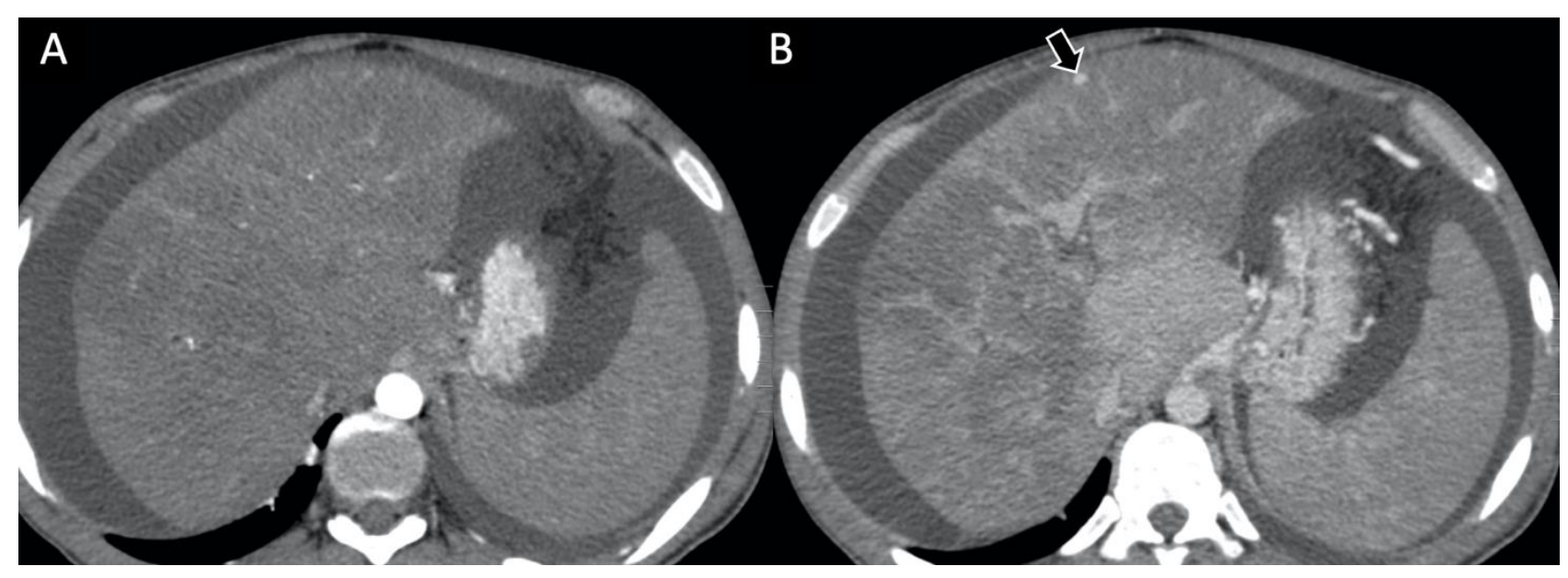

Fig. 13 - 29-year-old man with Budd-Chiari syndrome and idiopathic myelofibrosis. Axial CT image on (a) arterial and (b) portal venous phases demonstrate hepatomegaly, splenomegaly and heterogeneous hepatic and liver enhancement, with higher density of caudate lobe compared to the remaining liver on portal venous phase due to a different venous drainage. A small focal nodular hyperplasia-like lesion (arrow) is demonstrated in the left lobe. 


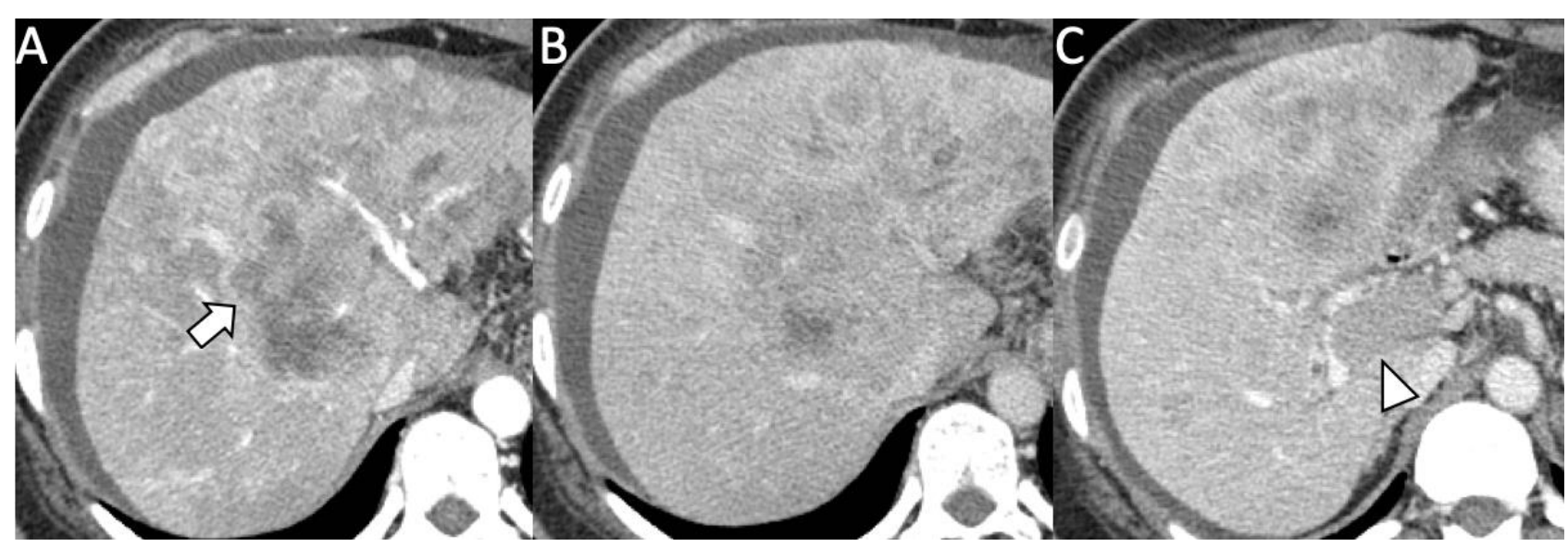

Fig. 14 - 47-year-old man with intrahepatic cholangiocarcinoma. Contrast enhanced CT image on (a) arterial phase shows a dominant mass (arrow) with peripheral enhancement, that (b) on portal venous phase demonstrates gradual centripetal central enhancement due to central fibrotic stroma. Other confluent satellite nodules are visible in the left liver. (c) Contrast enhanced CT images on portal venous phase at the level of the hepatic hilum shows the presence of tumor thrombus (arrowhead) in the portal vein, that is expanded by the tumor. 


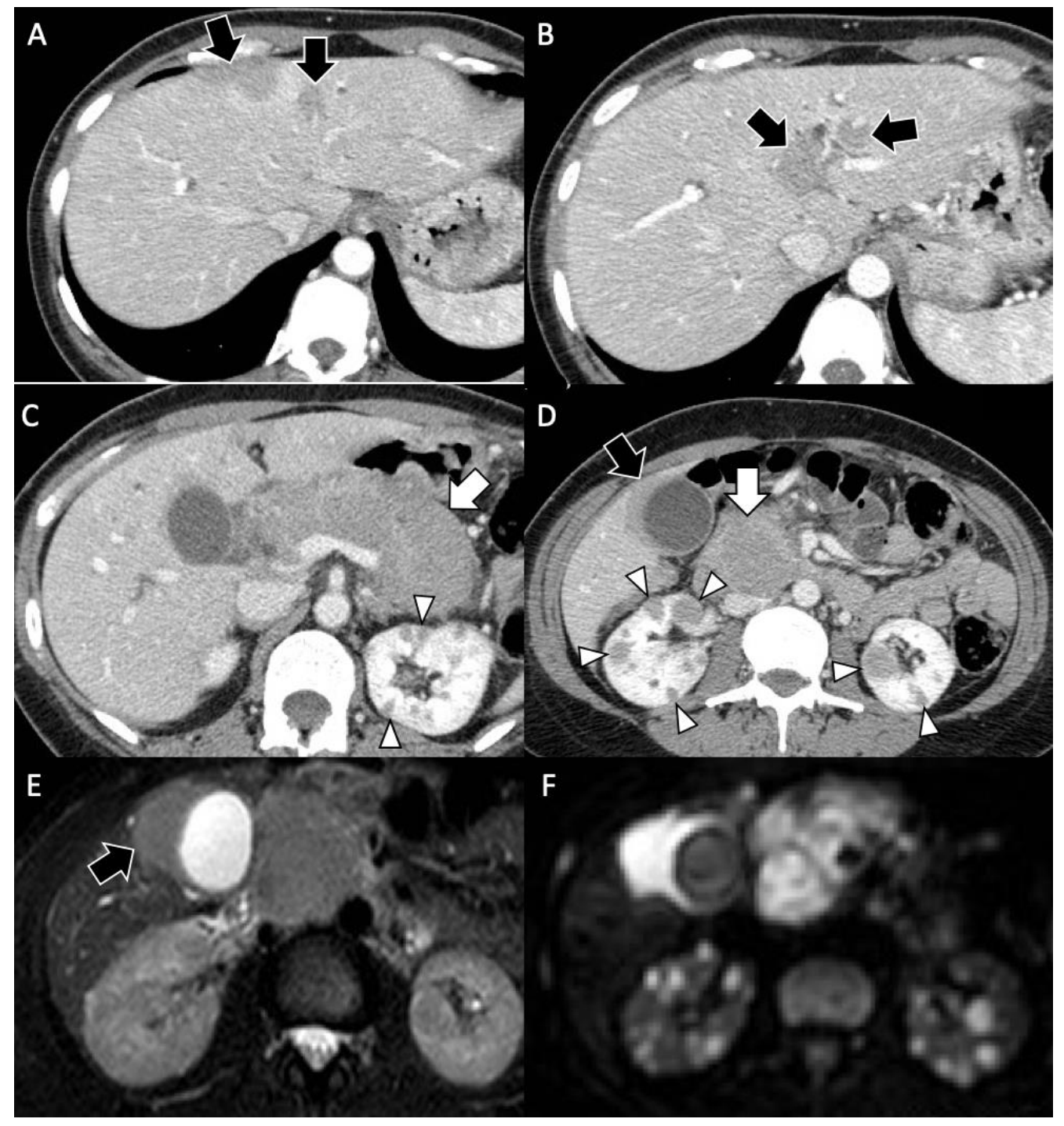

Fig. 15 - 54-year-old woman with B-cell lymphoma; symptoms at diagnosis included abdominal pain and unintentional weight loss (about 3 kilos in one week). Contrast enhanced CT images on $(a, b)$ arterial and $(c, d)$ portal venous phases show multiple hypoattenuating liver lesions (black arrows) alongside the portal branches, an enlarged and hypoattenuating pancreas (white arrow) as well as multiple bilateral tiny hypoattenuating renal lesions (arrowheads). Liver biopsy was performed and proved the diagnosis of B-cells lymphoma, morphologically consistent with Burkitt Lymphoma. MRI (e) on T2-weighted images show one of the lymphomatous lesions (arrow) slightly hyperintense on T2-weighted, with (f) restriction on diffusion weighted image. (f) Diffusion weighted image shows also diffusion restriction of the renal lesions. 


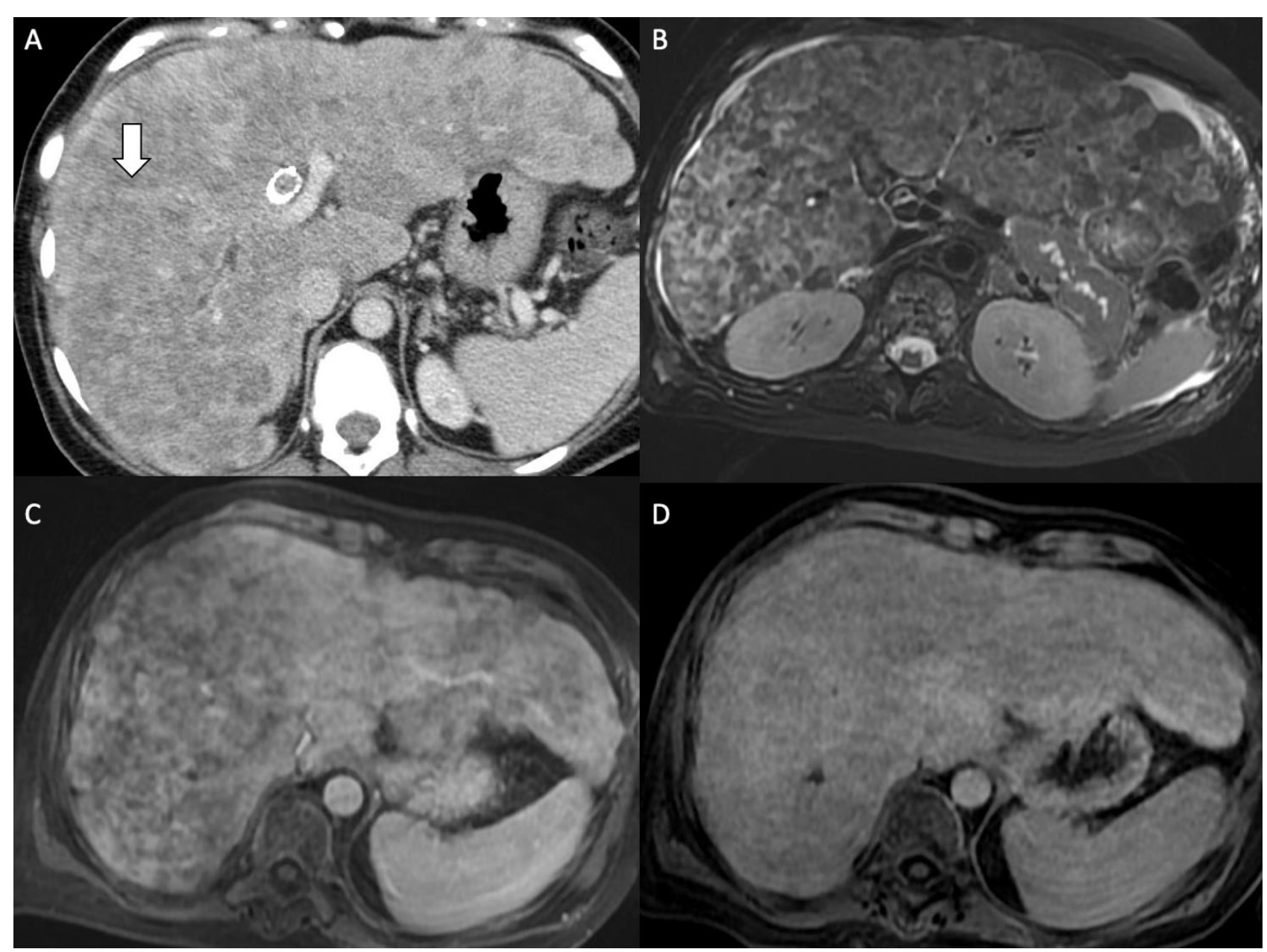

Fig. 16 - 48-year-old woman with breast carcinoma and pseudocirrhosis. (a) Contrast enhanced CT on portal venous phase shows multiple confluent hypoattenuating lesions surrounding portal vein branches (arrow) that are still patent and lobulated liver margins; the presence of multiple metastases may simulate infiltrative HCC. Gadoxetate enhanced MRI (b) on T2-weighted sequence shows a reticular pattern of hepatic parenchyma with slightly hyperintense lesions; a minimal ascites indicates worsened liver function. (c) On portal venous phase and (d) at 20 minutes after contrast injection liver parenchyma shows progressive contrast enhancement with lack of hypoattenuating lesions on hepatobiliary phase. Note isointensity of intrahepatic vessels to liver parenchyma due hepatic decompensation. 


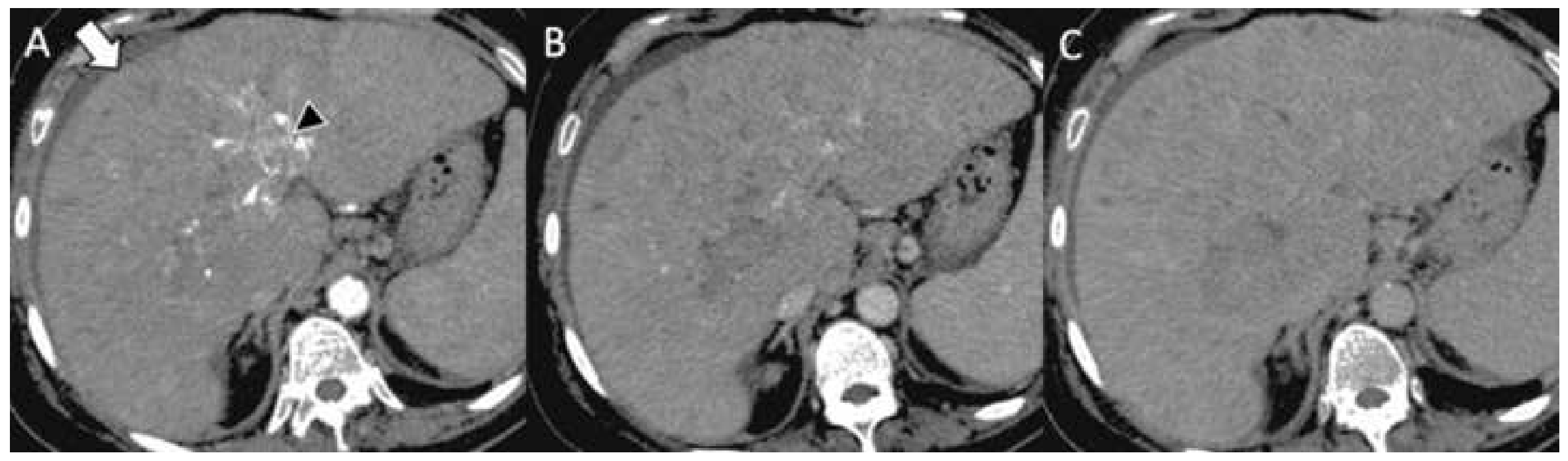




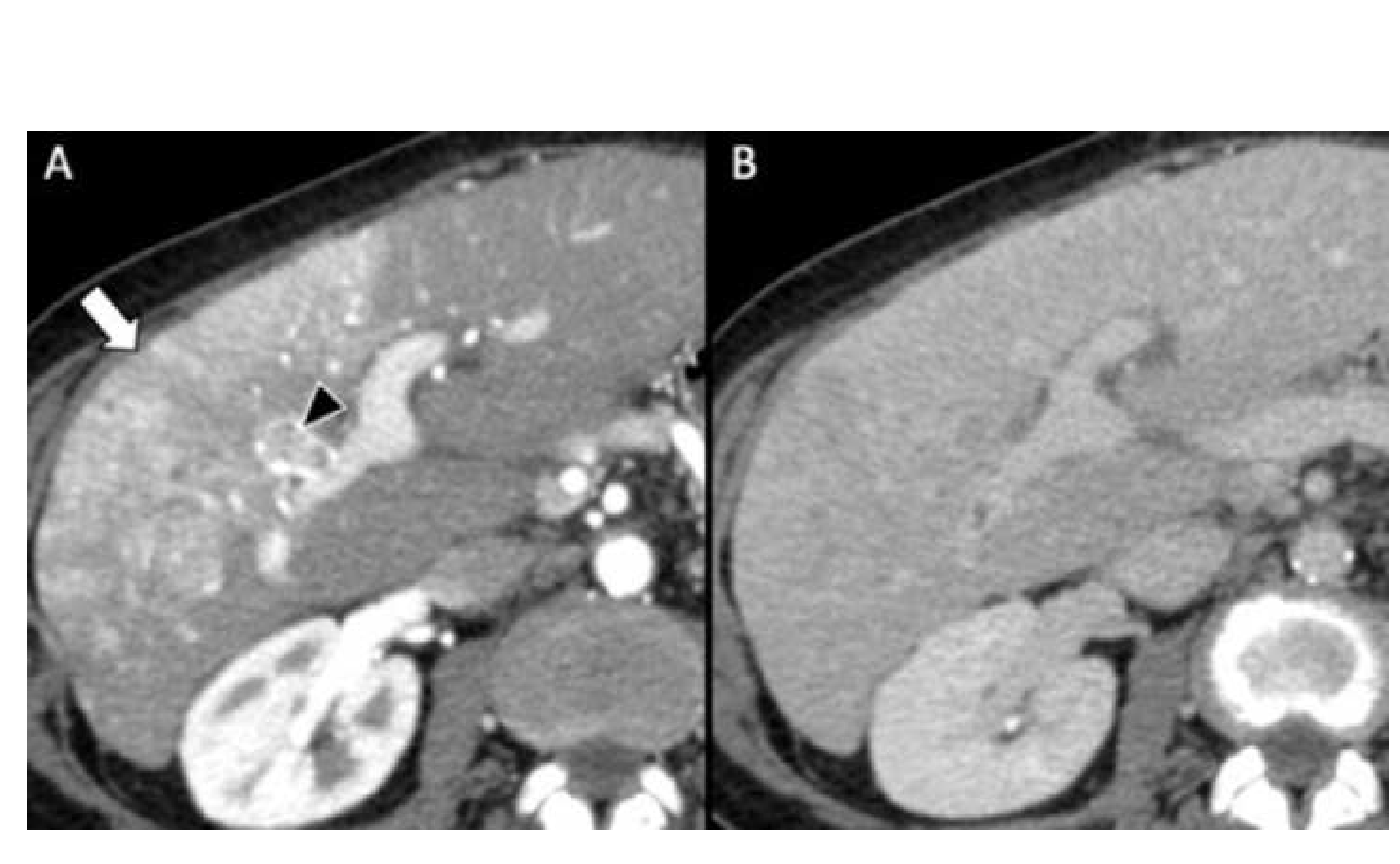

2




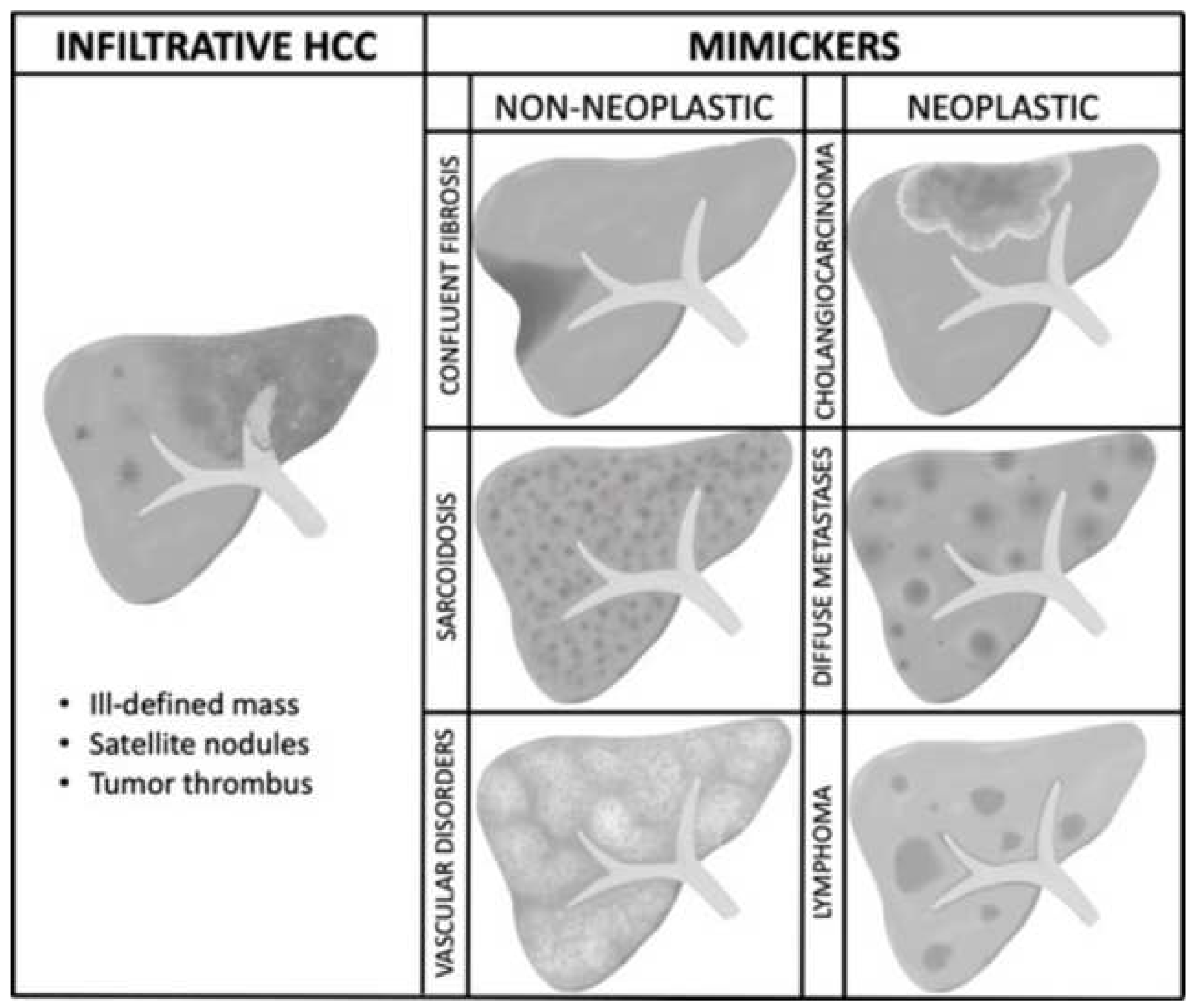




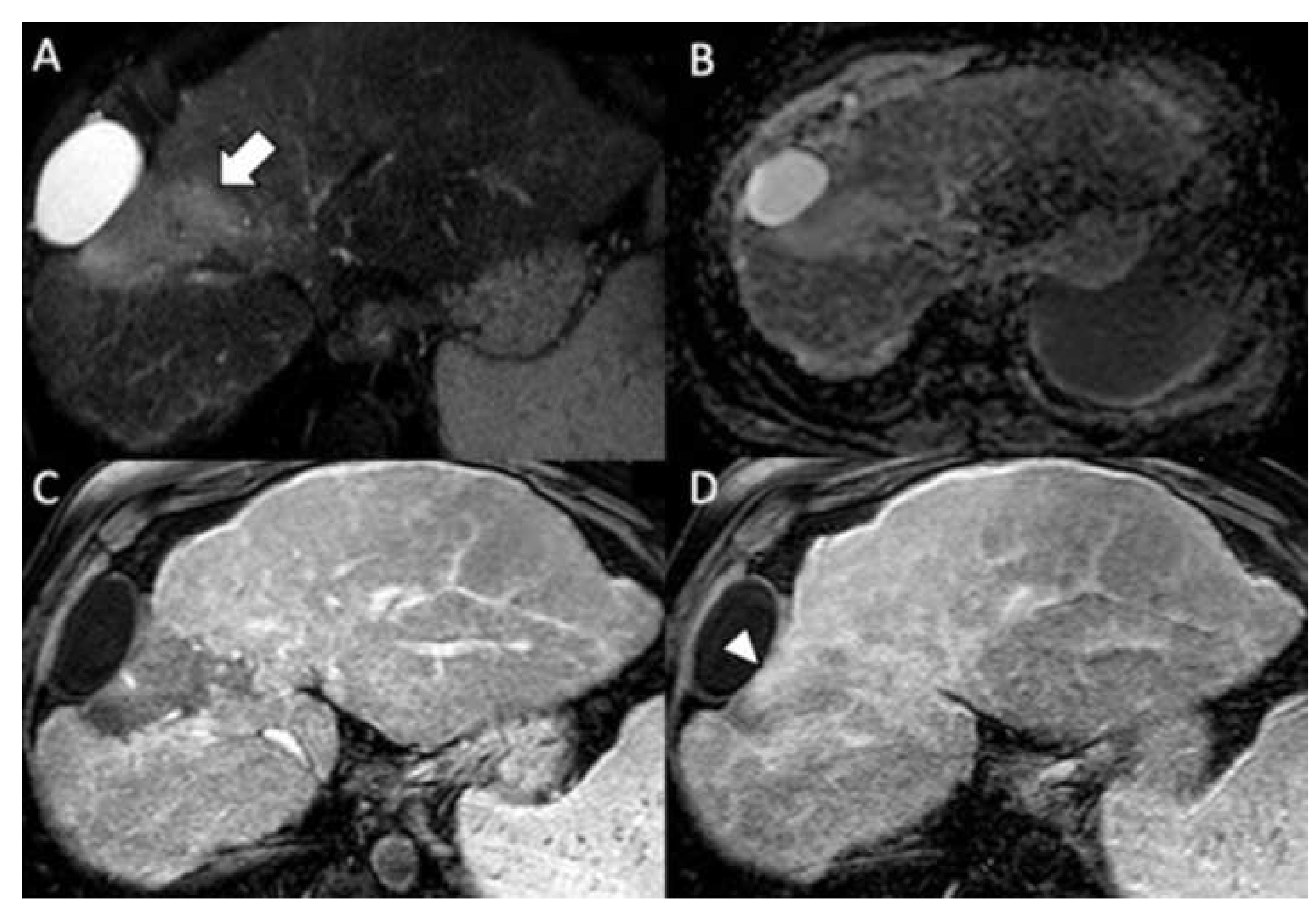




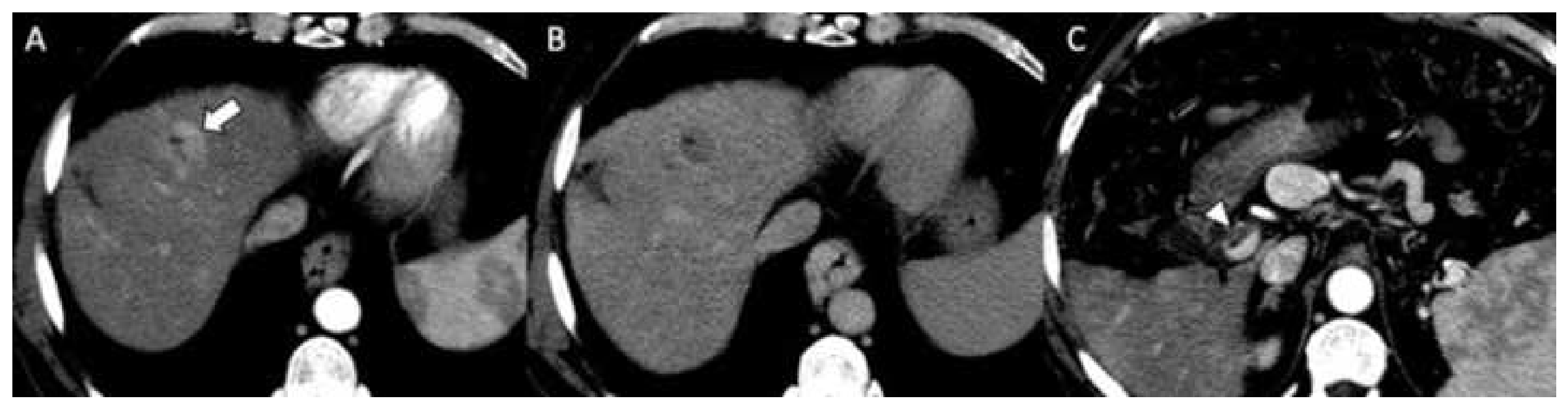




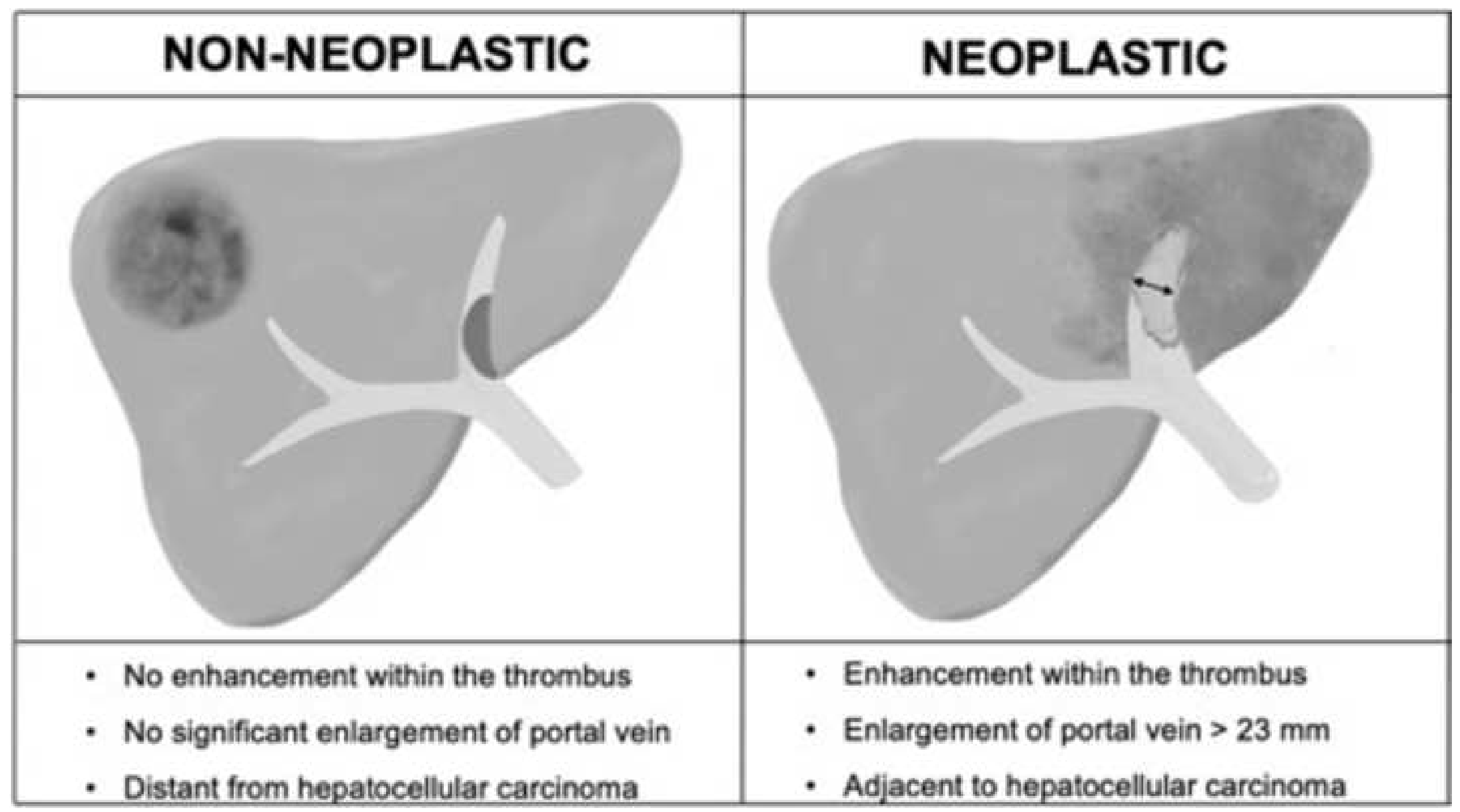




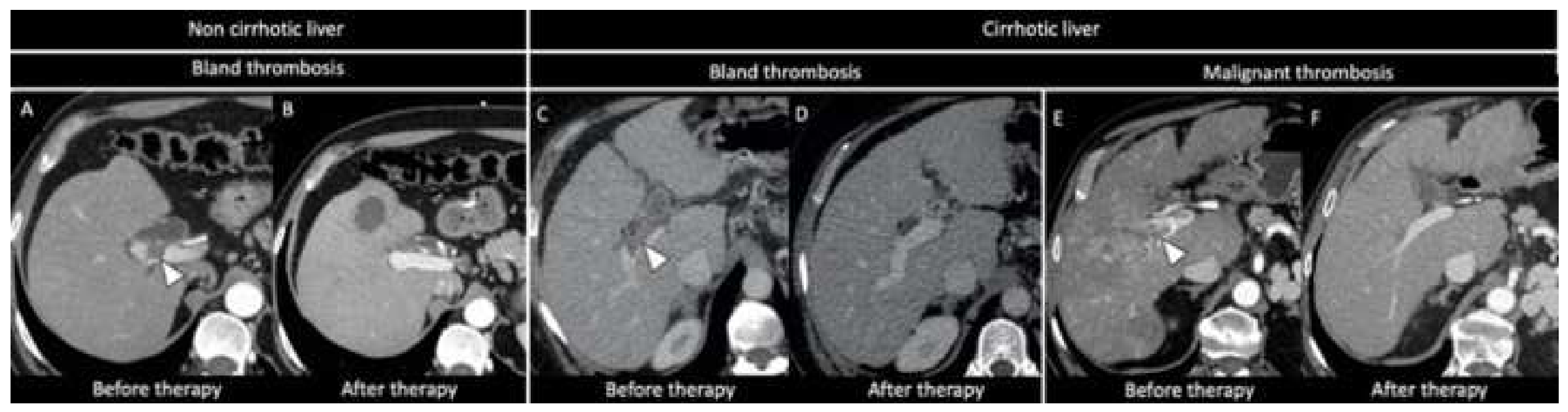




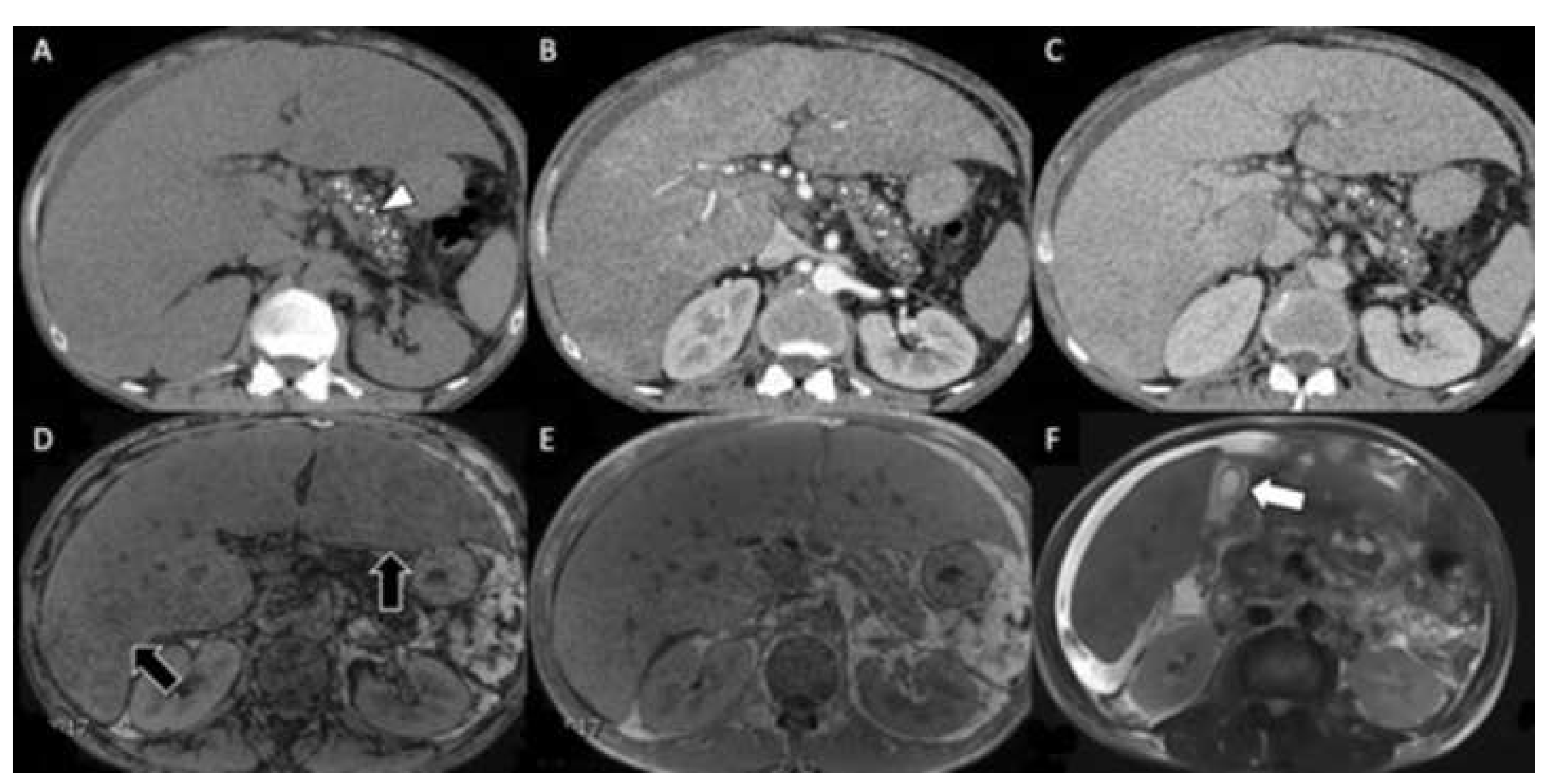




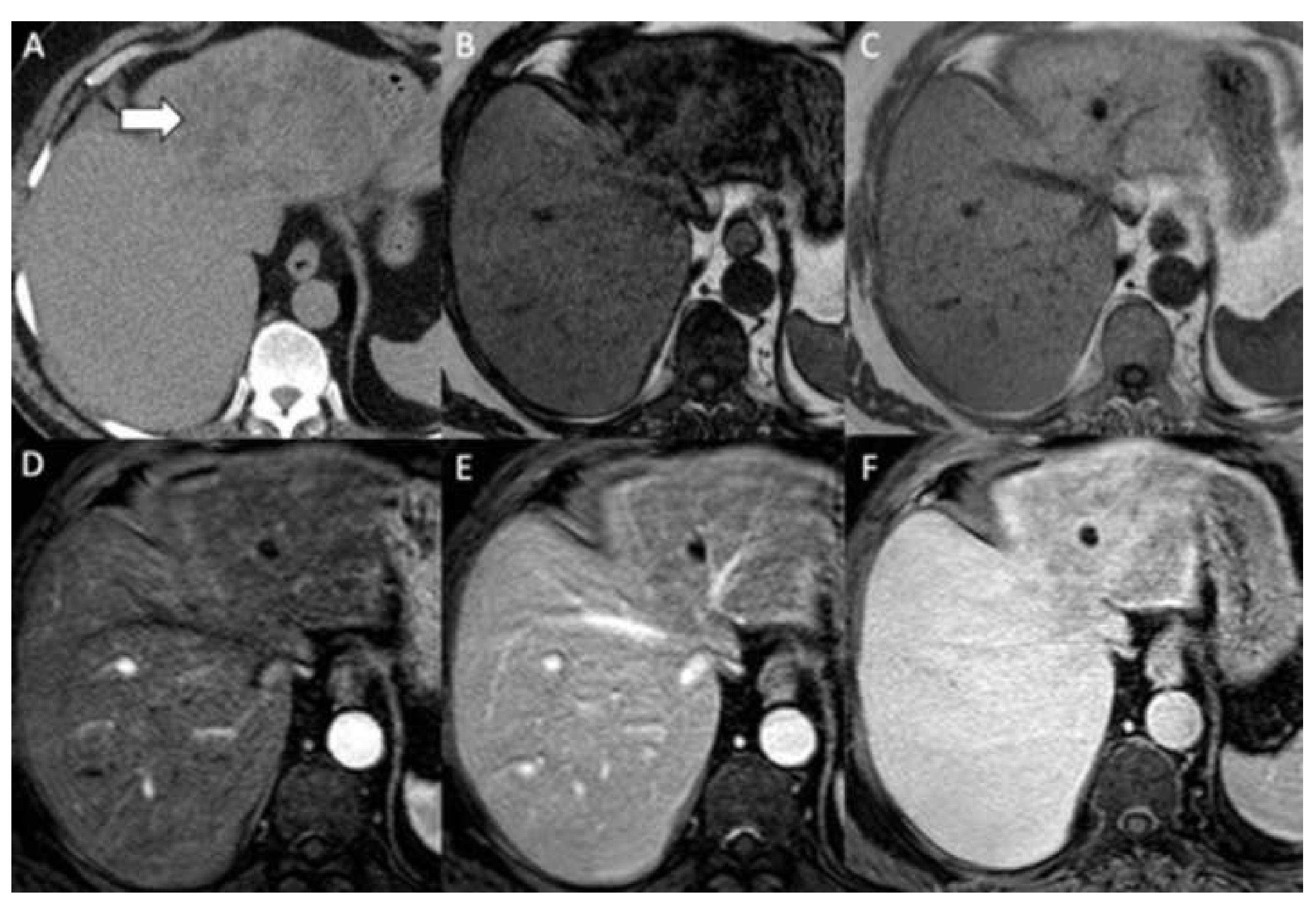



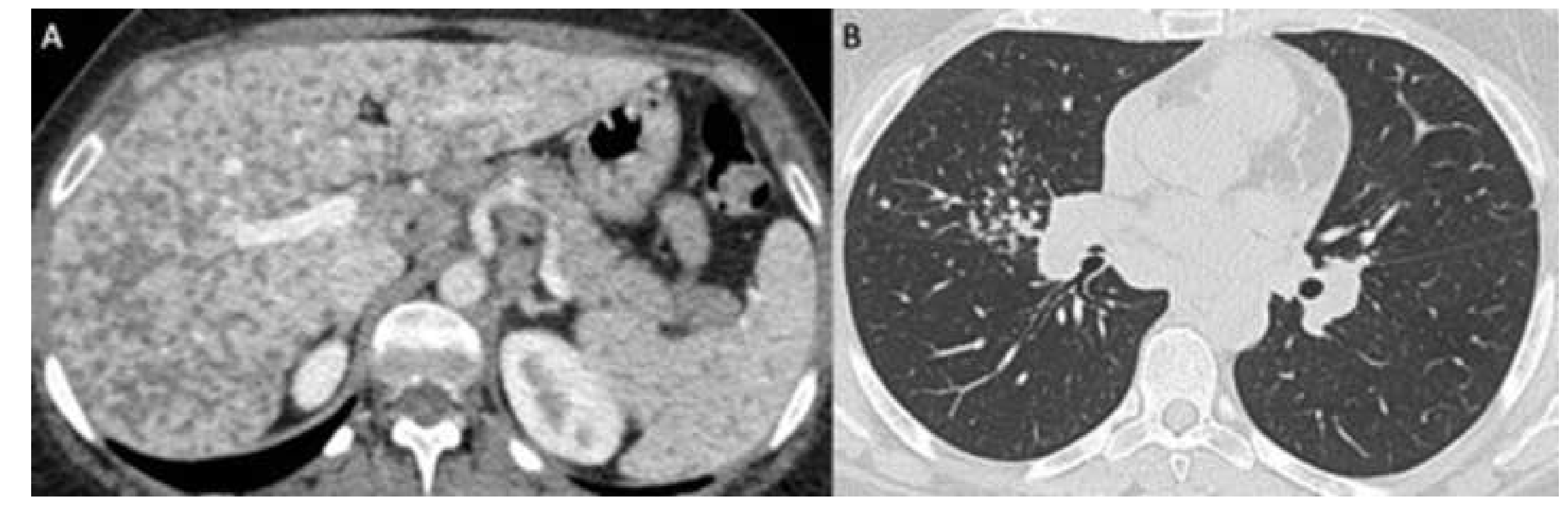


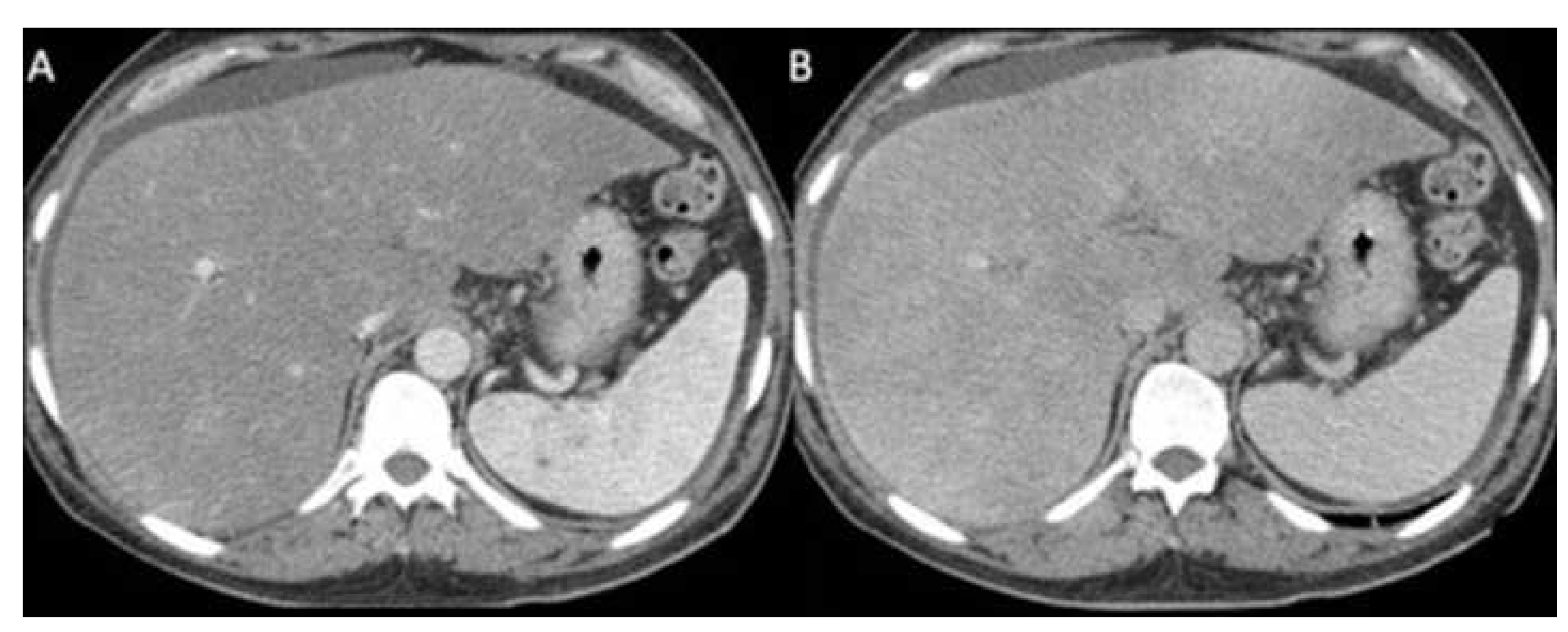



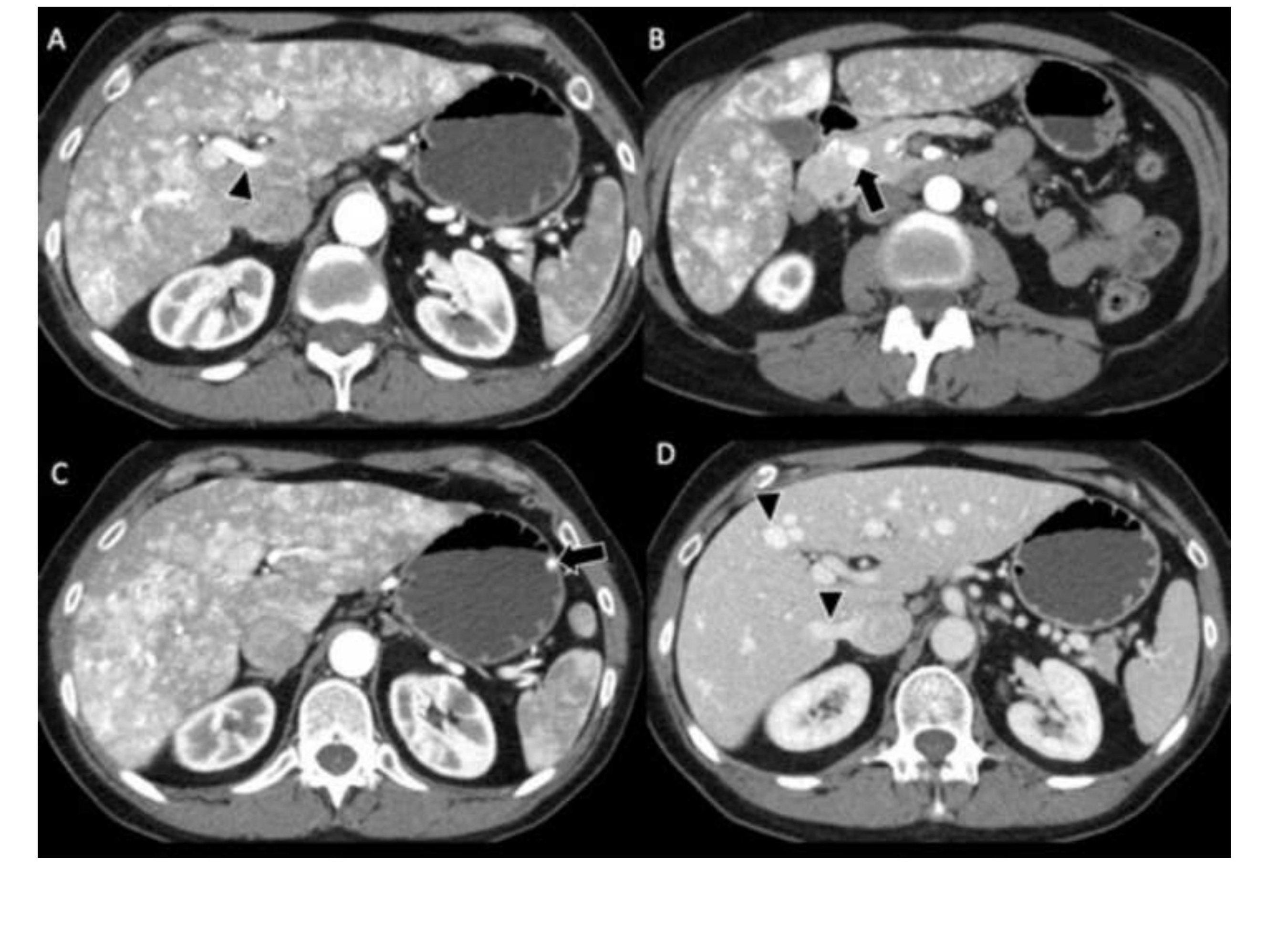


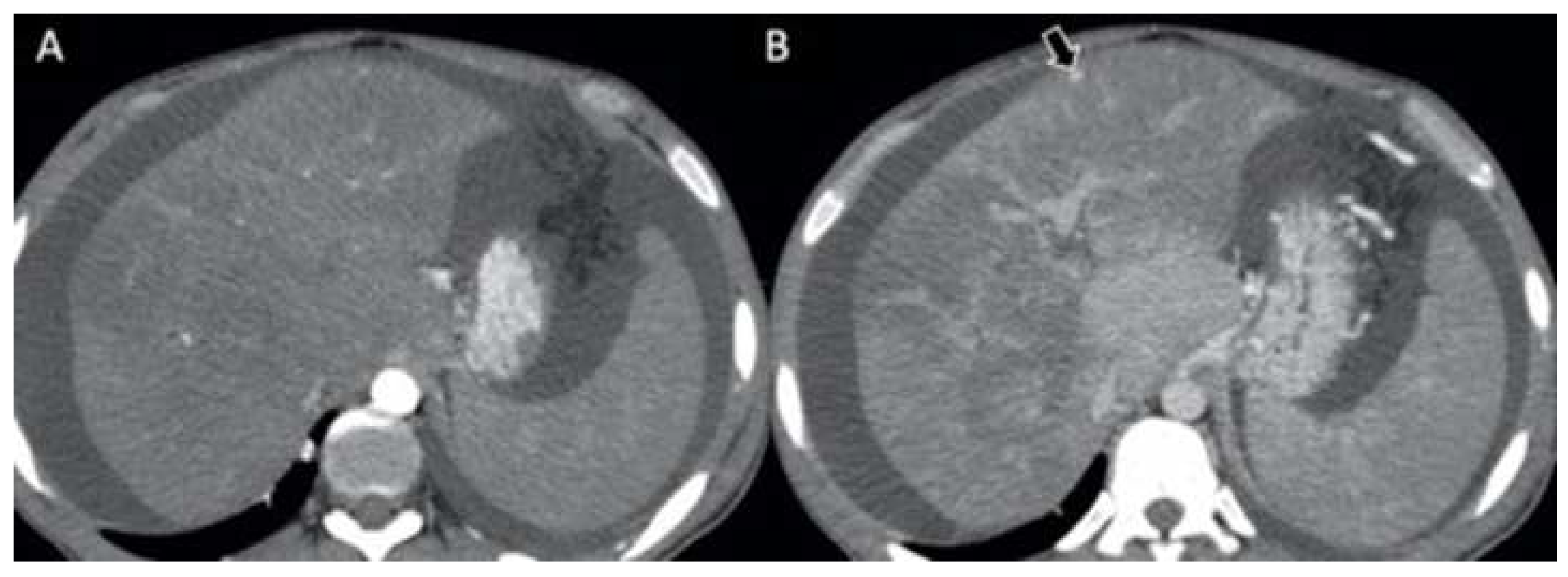




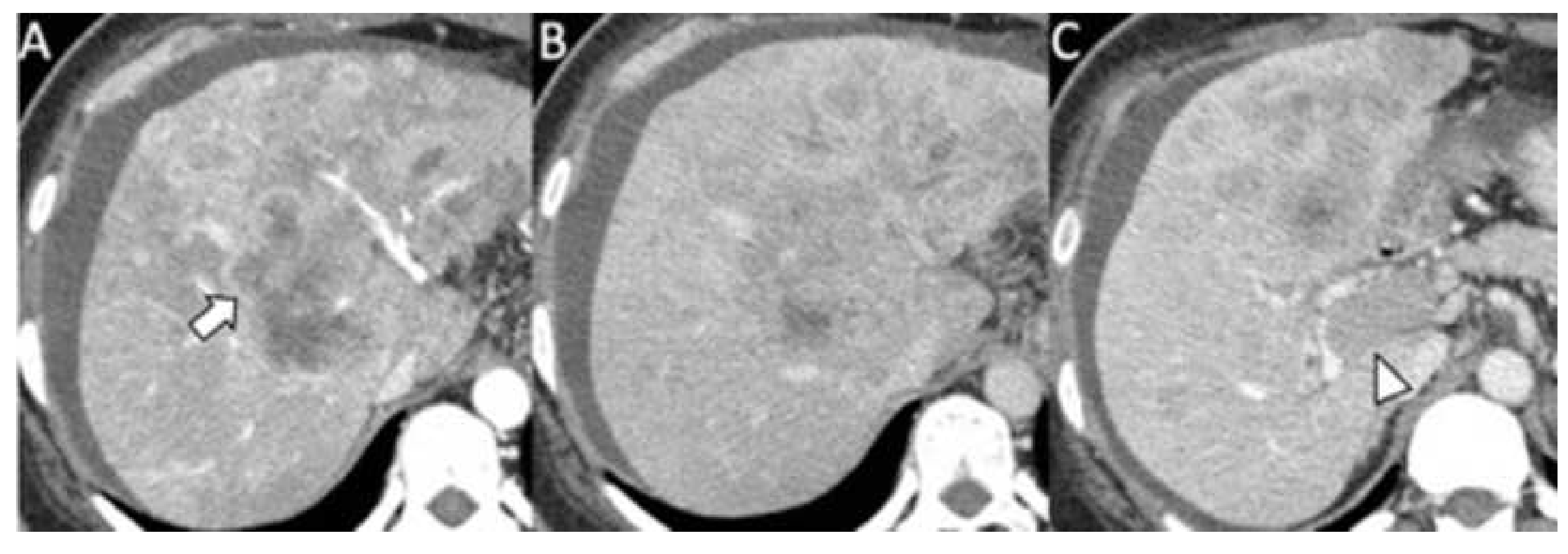


Click here to access/download;Figure;Picture15.jpg $\stackrel{\underline{ }}{ }$

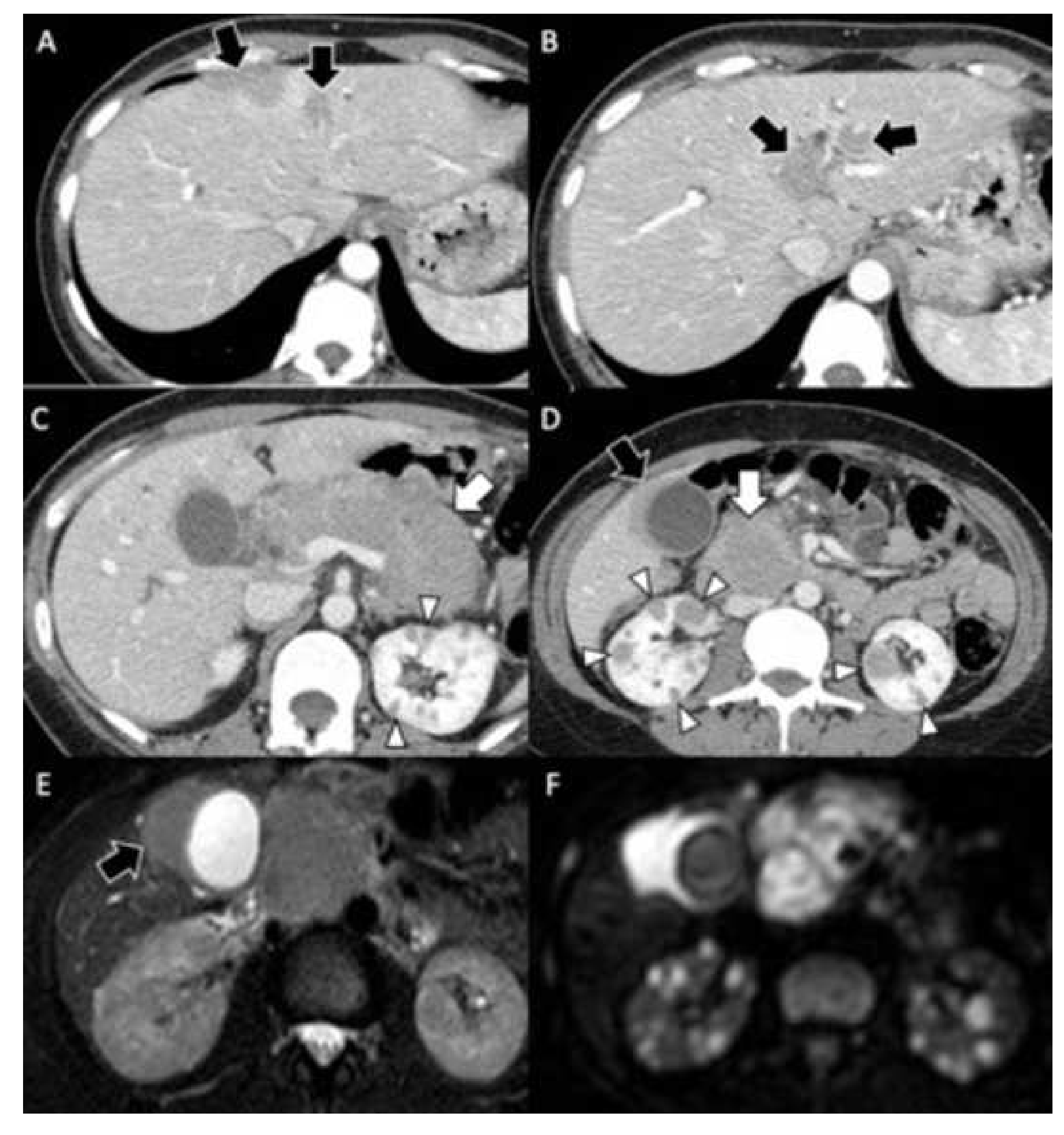

E

\section{.}


Click here to access/download;Figure;Picture16.jpg $\stackrel{\underline{ }}{ }$

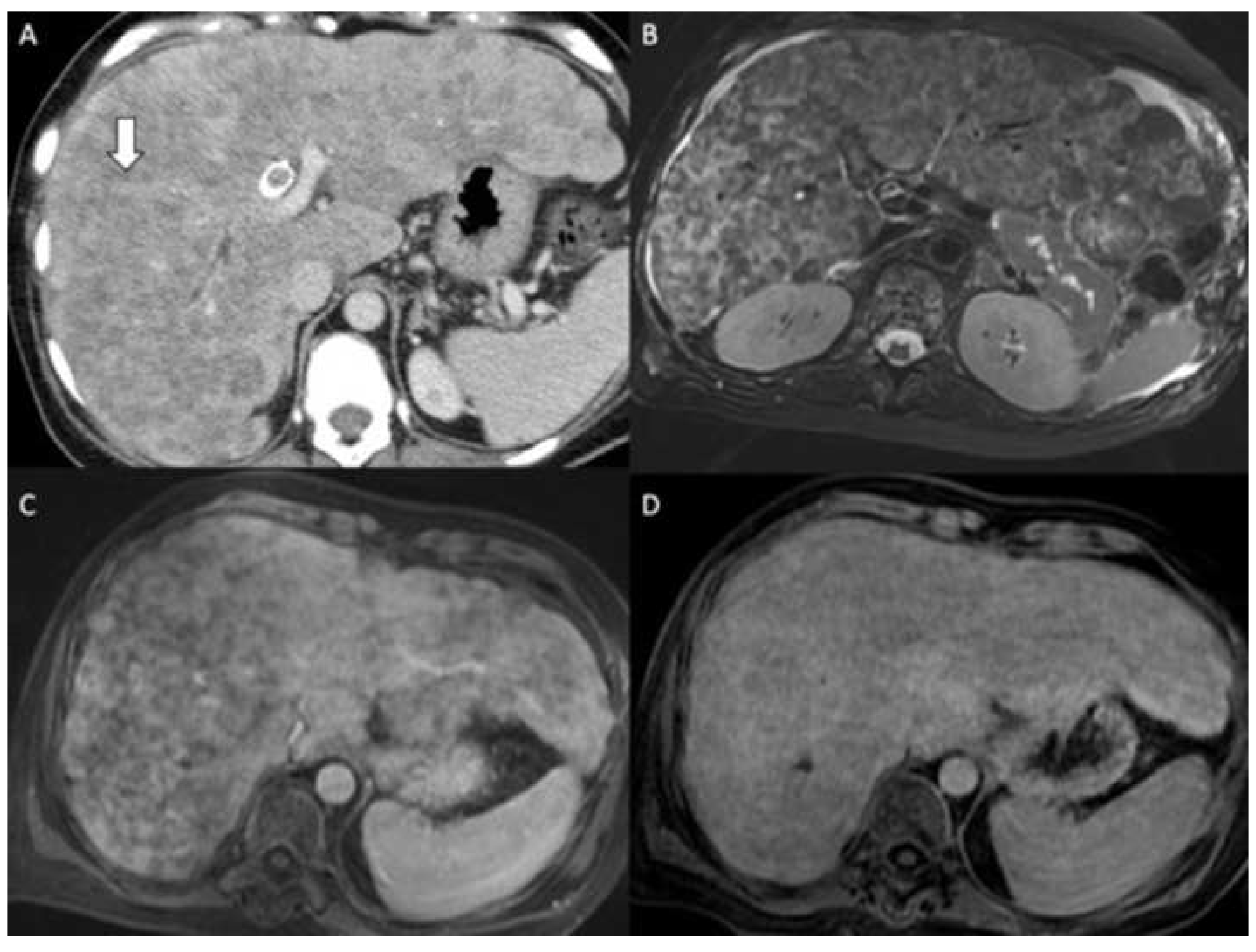

c
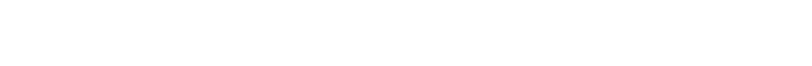


\section{Highlights}

- Infiltrative HCC appears as an ill-defined large mass with variable enhancement

- Portal vein tumor thrombosis appears as enhancing thrombus close to the main tumor

- Diagnosis of infiltrative HCC and neoplastic thrombosis may be challenging

- Many benign and malignant lesions may mimic infiltrative HCC and tumor thrombosis 